\title{
OVERDETERMINED SYSTEMS OF LINEAR PARTIAL DIFFERENTIAL EQUATIONS ${ }^{1}$
}

\author{
BY D. C. SPENCER
}

Introduction. The purpose of these lectures is to report on some recent developments in the theory of overdetermined systems of linear partial differential equations. The simplest, and most classical, example of an over-determined system is provided by the equations

$$
\frac{\partial f}{\partial x^{k}}=\phi_{k}, \quad k=1,2, \cdots, n,
$$

where the $\phi_{k}=\phi_{k}(x)$ are given (differentiable) functions, $f=f(x)$ is a function to be determined, and $x=\left(x^{1}, x^{2}, \cdots, x^{n}\right)$. The system is locally solvable for $f$ if and only if the compatibility conditions

$$
\frac{\partial \phi_{k}}{\partial x^{l}}=\frac{\partial \phi_{l}}{\partial x^{k}}, \quad k, l=1,2, \cdots, n,
$$

are satisfied. Write $\phi=\sum_{k=1}^{n} \phi_{k} d x^{k}$ and introduce the differential operator $d$ where

$$
d f=\sum_{k=1}^{n}\left(\partial f / \partial x^{k}\right) d x^{k}
$$

then the above system can be written in the form $d f=\phi$ and the compatibility condition becomes simply $d \phi=0$ where $d$ here denotes the usual exterior differential operator

$$
d \phi=\sum_{k<l}\left(\partial \phi_{l} / \partial x^{k}-\partial \phi_{k} / \partial x^{l}\right) d x^{k} \wedge d x^{l} .
$$

We are thus led to introduce the following complex (initial portion of the de Rham complex)

$$
A^{0} \stackrel{d}{\rightarrow} A^{1} \stackrel{d}{\rightarrow} A^{2}
$$

where $A^{0}$ is the sheaf of germs of (differentiable) functions, $A^{1}$ and $A^{2}$ are the sheaves of germs of differential forms of degrees 1 and 2 respectively. The exactness of the complex expresses the local solvability of the overdetermined system under the required compatibility conditions.

1 Research supported by a National Science Foundation Grant, GN-530, to the American Mathematical Society. Received by the editors September 13, 1968. 
In general, an overdetermined system of linear partial differential equations can be regarded as a complex

$$
E \stackrel{D}{\longrightarrow} F \stackrel{D_{1}}{\longrightarrow} G
$$

where $E, F$ and $G$ are sheaves of germs of differentiable sections of vector bundles over a differentiable manifold $X$ and $D, D_{1}$ are differential operators. (In the above simple example, $A^{0}$ is the sheaf of sections of the trivial bundle $X \times R$, where $R$ denotes the real numbers, and $A^{1}, A^{2}$ are respectively the sheaves of sections of $T^{*}$, $\Lambda^{2} T^{*}$ where $T^{*}$ is the cotangent bundle of $X$ and $\Lambda^{2} T^{*}$ the exterior product.) The basic problem, in the case where the complex is assumed to be of constant rank and formally exact (in a sense to be made precise later), is to find reasonable sufficient conditions in order that such a complex be exact. The condition currently receiving the most attention is that the complex be elliptic, but even under this hypothesis the exactness is not proved in general. It is conceivable, but not widely accepted, that ellipticity may not be the right condition.

The first step in the study of overdetermined systems is obviously the investigation of the formal properties of the given complex and the finding of criteria in order that the complex be formally exact. This we call the formal theory. The formal theory has now been brought into satisfactory shape, largely by the foundational work of D. G. Quillen [19] and H. Goldschmidt [6] which is based on an earlier approach of the author [21] (numbers in brackets refer to the references listed at the end). Recently, Goldschmidt (loc. cit.) has given a definitive treatment of some of the main aspects of the formal theory. Highly significant contributions to the formal theory have also been made by V. W. Guillemin, M. Kuranishi and S. Sternberg (see [8], [9], [10], [14]). Indeed, the recent development of the formal theory owes much to the pioneer work carried out by Kuranishi during the 1950 's. A fairly complete summary of the formal theory, from the point of view of these authors, is given in the first part of this report. Lack of space, however, has forced us to neglect the significant contributions of B. Malgrange [17], in particular the dual point of view adopted in his paper [17(a)] in which he looks at differential operators rather than defining equations and formal solutions. Other approaches to the formal theory are necessarily omitted, for example the development given by Ngô Van Quê [24] which is adapted to structures on manifolds. 
As a biproduct of the formal theory, a much more satisfactory formulation of the Cartan-Kähler theorem is obtained in the analytic case.

The second, and harder, step in the study of overdetermined systems is the determination of satisfactory criteria for the exactness, in the differentiable sense, of the given complex (assumed to be formally exact), and we call this the existence theory (in the differentiable case). The example of $\mathrm{H}$. Lewy [15], which is even a determined system with compatibility operator $D_{1}$ equal to zero, shows us that exactness in the differentiable sense is not always true. The second part of this report is an incomplete summary of the existence theory (in the differentiable case), and much of the best and most significant work is either ignored or mentioned very briefly. The main content of the second part is concerned with a homological criterion ( $\delta$-estimate) which B. MacKichan [16] has recently shown to be a reasonable generalization to overdetermined systems of the notion of "uncoupled" (or "diagonal") operators. The significance of this estimate was first recognized by I. M. Singer. In particular, if the system is elliptic, the $\delta$-estimate implies the solvability of the Neumann problem on domains satisfying appropriate convexity conditions (see \$2.3).

Since an adequate treatment would fall outside the scope and space requirements of this report, the work of L. Hörmander (for example, [11(c)]) is not described nor is recent related work of M. Kuranishi.

It seems possible that future significant developments of the theory may center around a study of the characteristic variety of the differential operator, bringing to bear on these developments all the powerful machinery of modern algebraic geometry. A beginning in this direction has been made by V. W. Guillemin and S. Sternbergfor example, their theorem on the integrability of the characteristic variety. Closely related to this is the investigation, from an algebraic point of view, of the ring of formal differential operators and its quotient field of formal pseudodifferential operators. But it is too early to say much about these matters here.

\section{Formal theory.}

1.1. Jets. Let $X$ be a differentiable manifold of dimension $n$, where "differentiable" here and elsewhere means "differentiable of class $C^{\infty}$." If $E$ is a (differentiable) vector bundle over $X$ we denote, for each nonnegative integer $k$, by $J_{k}(E)$ the vector bundle over $X$ of $k$-jets of $E$. The fibre of $J_{k}(E)$ over a point $x$ of $X$ is the quotient of 
the space of germs of sections of $E$ at $x$ by the subspace of germs which vanish to order $k+1$ at $x$. We identify $J_{0}(E)$ with $E$, and denote by $\pi: J_{k}(E) \rightarrow X, \pi_{k-1}: J_{k}(E) \rightarrow J_{k-1}(E)$, the natural projections. If $E$ is the trivial line bundle, we write $J_{k}$ for $J_{k}(E)$ where $J_{k}$ is the vector bundle of $k$-jets of differentiable functions.

We denote by $T$ the tangent bundle of $X$, by $T^{*}$ the cotangent bundle of $X$, and by $S^{k} T^{*}, \Lambda^{l} T^{*}$ and $\otimes^{m} T^{*}$ the $k$-tuple symmetric product of $T^{*}$, the $l$-tuple exterior product of $T^{*}$ and the $m$-tuple tensor product of $T^{*}$, respectively. There is a natural vector-bundle morphism $\epsilon: S^{k} T^{*} \otimes E \rightarrow J_{k}(E)$, and the sequence

$$
0 \longrightarrow S^{k} T^{*} \otimes E \stackrel{\epsilon}{\longrightarrow} J_{k}(E) \stackrel{\pi_{k-1}}{\longrightarrow} J_{k-1}(E) \longrightarrow 0
$$

is exact.

If $E$ is a vector bundle we shall, for simplicity of notation, also write $E$ for the sheaf of germs of differentiable sections of $E$. The interpretation of $E$ as a sheaf of germs will only occur in contexts where differentiation is involved and such occurrences will always be clear. We write $\theta$ for the sheaf of germs of differentiable functions (structure sheaf of $X$ ).

It will sometimes be convenient to regard jets from the point of view adopted by $B$. Malgrange $[17(a)]$, and based on ideas of $A$. Grothendieck [7] (see also H. Cartan [3]). Namely, let $Y$ be another copy of $X$, let $\hat{\theta}_{X \times Y}$ be the structure sheaf of $X \times Y$ and $\mathfrak{J}$ the ideal of $\mathcal{O}_{X \times Y}$ vanishing on the diagonal $\triangle$ of $X \times Y$ (defining ideal of $\triangle$ ). Then $J_{k}$ can be identified with the quotient $\hat{\theta}_{X \times Y} / J^{k+1}$. Let $i: \mathcal{O} \rightarrow \mathcal{O}_{X \times Y}$ be the map induced by the projection $\pi_{1}: X \times Y \rightarrow X$, i.e., $i=\pi_{1}^{*}$. Let $r: X \times Y \rightarrow X \times Y$ be reflection on the diagonal $\triangle$, and let $j=\left(\pi_{1} \circ r\right)^{*}$. By passage to the quotient we obtain from $i, j$, respectively, the maps $i_{k}, j_{k}: \mathcal{O} \rightarrow J_{k}$ where $j_{k}$ associates to a function $f$, and the point $x$, the $k$-jet of $f$ at $x$, and $i_{k}$ associates to it the constant function $f(x)$. The maps $i_{k}$ and $j_{k}$ impart to $J_{k}$ two structures of $\theta$ modules; namely, structures of left and right $\theta$-modules, respectively. In a tensor product of (sheaves of) $\mathcal{O}$-modules with $J_{k}$ as factor, the position of $J_{k}$ indicates the structure to be considered. For example, if $A$ is a sheaf of $\mathcal{O}$-modules, $J_{k} \otimes_{\mathcal{O}} A$ is the tensor product of $J_{k}$ and $A$ where $J_{k}$ is regarded as a right $\theta$-module and the product itself inherits a structure of left module from the left structure of $J_{k}$. Write $J_{k}(A)=J_{k} \otimes_{\ominus} A$. Let $j_{k}: A \rightarrow J_{k}(A)$ be the map sending a germ $a$ of a section of $A$ into $j_{k}(a)=j_{k}(1) \otimes a$ and therefore, if $f$ is a germ of function, sending $f \cdot a$ into $j_{k}(f \cdot a)=j_{k}(1) \otimes f a=j_{k}(f) \otimes a$. In the case where $A=E$, the sheaf of germs of sections of a vector bundle $E$, we 
have $J_{k}(A)=J_{k}(E)$, the sheaf of germs of sections of $J_{k}(E)$ (the bundle of $k$-jets of $E$ ).

1.2. Differential operators and their prolongations. Let $E, F$ be vector bundles over $X$, and let

$$
\phi: J_{k}(E) \rightarrow F
$$

be a morphism of vector bundles. Then $\phi$ induces a sheaf morphism $\phi: J_{k}(E) \rightarrow F$ (for which we use the same letter).

Definition 1.2.1. The symbol $\sigma(\phi)$ of $\phi$ is the composition

$$
\sigma(\phi)=\phi \circ \epsilon: S^{k} T^{*} \otimes E \rightarrow F .
$$

Definition 1.2.2. A sheaf morphism

$$
D: E \rightarrow F
$$

is called a differential operator (from $E$ to $F$ ) of order $k$ if the triangle

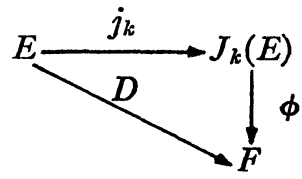

commutes, i.e., if $D=\phi \circ j_{k}$, where $\phi: J_{k}(E) \rightarrow F$ is a bundle morphism. The symbol $\sigma(D)$ of $D$ is the symbol of $\phi$, i.e., $\sigma(D)=\sigma(\phi)$.

Since there is a one-one correspondence between differential operators $D: E \rightarrow F$ of order $k$ and bundle morphisms $\phi: J_{k}(E) \rightarrow F$, we shall also call a morphism $\phi$ a differential operator.

Definition 1.2.3. The $l$ th prolongation $p_{l}(\phi): J_{k+l}(E) \rightarrow J_{l}(F)$ of $\phi$ is the unique morphism of vector bundles such that the following diagram commutes:

$$
\begin{aligned}
& J_{k+l}(E) \stackrel{p_{l}(\phi)}{\longrightarrow} J_{l}(F)
\end{aligned}
$$

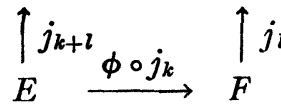

The differential operator $D_{l}=j_{l} \circ \phi \circ j_{k}: E \rightarrow J_{l}(F)$ is the $l$ th prolongation of the operator $D=\phi \circ j_{k}: E \rightarrow F$. We shall sometimes write $p_{l}(D)=p_{l}(\phi)$ and $p(D)=p_{0}(\phi)=\phi$.

In particular, let $1_{k}: J_{k}(E) \rightarrow J_{k}(E)$ be the identity map (the corresponding differential operator is then $j_{k}: E \rightarrow J_{k}(E)$ ). The $l$ th prolongation of the map $1_{k}$ is a monomorphism of vector bundles 


$$
p_{l}\left(1_{k}\right): J_{k+l}(E) \rightarrow J_{l}\left(J_{k}(E)\right)
$$

and we identify $J_{k+l}(E)$ with its image in $J_{l}\left(J_{k}(E)\right)$. More generally, for each pair of nonnegative integers $k, l$, we can identify $J_{l}\left(J_{k}(E)\right.$ ) with its image in $J_{1}^{k+l}(E)$, where $J_{1}^{k+l}$ is the $(k+l)$ th iterate of $J_{1}$.

LEMмA 1.2.1 (QUILLEN [19]). For $k \geqq 0, l \geqq 1$, we have

$$
J_{k+l}(E)=J_{l}\left(J_{k}(E)\right) \cap J_{l-1}\left(J_{k+1}(E)\right)
$$

where $J_{k+l}(E), J_{l}\left(J_{k}(E)\right)$ and $J_{l-1}\left(J_{k+1}(E)\right)$ are regarded as sub-bundles of $J_{1}^{k+l}(E)$.

The proof of this lemma is left as an exercise.

Definition 1.2.4. The map $J_{l}(\phi): J_{l}\left(J_{k}(E)\right) \rightarrow J_{l}(F)$ is the morphism of vector bundles such that the following diagram commutes:

$$
\begin{aligned}
& J_{l}\left(J_{k}(E)\right) \stackrel{J_{l}(\phi)}{\longrightarrow} J_{l}(F) \\
& \begin{array}{rll}
\uparrow j_{l} & & \uparrow j_{l} \\
J_{k}(E) \stackrel{\phi}{\longrightarrow} & F
\end{array}
\end{aligned}
$$

The symbol of $J_{l}(\phi)$ is the composition

$$
S^{l} T^{*} \otimes J_{k}(E) \stackrel{\phi}{\rightarrow} S^{l} T^{*} \otimes F \stackrel{\epsilon}{\rightarrow} J_{l}(F) .
$$

Lemma 1.2.2. Let $\phi: J_{k}(E) \rightarrow F$ be a morphism, let $p_{l+m}(\phi): J_{k+l+m}(E)$ $\rightarrow J_{l+m}(F)$ be its $(l+m)$ th prolongation, and let $J_{m}\left(p_{l}(\phi)\right): J_{m}\left(J_{k+l}(E)\right)$ $\rightarrow J_{m}\left(J_{l}(F)\right)$ be the map obtained by applying the functor $J_{m}$ to the bundle map $p_{l}(\phi): J_{k+l}(E) \rightarrow J_{l}(F)$. Then the following diagram is commutative:

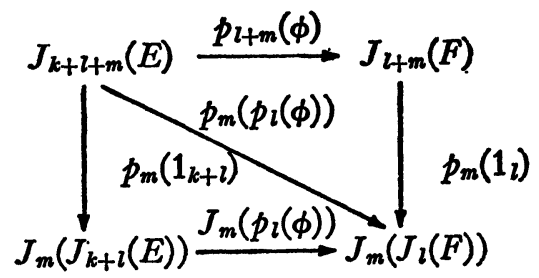

The proof is by diagram chasing, and we omit it (see Goldschmidt [6(b)]).

For $l \geqq 1$ we define $\sigma_{l}(\phi): S^{k+l} T^{*} \otimes E \rightarrow S^{l} T^{*} \otimes F$ to be the morphism of vector bundles such that the following diagram is exact and commutative: 


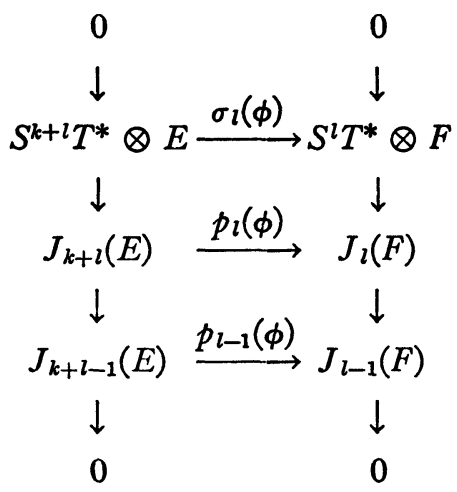

We set $\sigma_{0}(\phi)=\sigma(\phi)$.

Definition 1.2.5. A homogeneous linear partial differential equation $R_{k}$ of order $k$ on $E$ is a sub-bundle of $J_{k}(E)$. A solution of $R_{k}$ is a section $e$ of $E$ over an open set $U \subset X$ such that $j_{k}(e)(x) \in R_{k}$ for all $x \in U$. The $l$ th prolongation of $R_{k}$ is the subset $R_{k+l}=J_{l}\left(R_{k}\right) \cap J_{k+l}(E)$ of $J_{k+l}(E)$ where $J_{l}\left(R_{k}\right)$ is regarded as a subset of $J_{l}\left(J_{k}(E)\right)$. If $\phi: J_{k}(E) \rightarrow F$ is a bundle morphism of locally constant rank with $R_{k}$ as kernel, we say that $R_{k}$ is the equation associated with the differential operator $\phi \circ j_{k}: E \rightarrow F$. (Conversely, given a sub-bundle $R_{k}$ of $J_{k}(E)$, a vector bundle $F$ and morphism $\phi: J_{k}(E) \rightarrow F$ clearly exist with kernel $R_{k}$.) We set $R_{k-l}=J_{k-l}(E)$ for $1 \leqq l \leqq k$.

It is easily seen that $R_{k+l}$ is the kernel of $p_{l}(\phi)$, i.e.,

$$
0 \longrightarrow R_{k+l} \longrightarrow J_{k+l}(E) \stackrel{p_{l}(\phi)}{\longrightarrow} J_{l}(F) .
$$

Let $g_{k+l} \subset S^{k+l} T^{*} \otimes E$ be the kernel of the map $\pi_{k+l-1}: R_{k+l} \rightarrow R_{k+l-1}$, i.e., for $l \geqq 0$,

$$
0 \longrightarrow g_{k+l} \longrightarrow R_{k+l} \stackrel{\pi_{k+l-1}}{\longrightarrow} R_{k+l-1} .
$$

Then $g_{k+l}$ is also the kernel of $\sigma_{l}(\phi): S^{k+l} T^{*} \otimes E \rightarrow S^{l} T^{*} \otimes F$, i.e.,

$$
0 \longrightarrow g_{k+l} \longrightarrow S^{k+l} T^{*} \otimes E \stackrel{\sigma_{l}(\phi)}{\longrightarrow} S^{l} T^{*} \otimes F .
$$

Definition 1.2.6. We call $g_{k}$ the symbol of the equation $R_{k}$ and set $g_{k-l}=S^{k-l} T^{*} \otimes E$ for $1 \leqq l \leqq k$.

It is important to note that $R_{k+l}$ and $g_{k+l}$ are families of vector spaces over $X$ and are not necessarily vector bundles for $l \geqq 0$ (with the exception of $R_{k}$, a vector bundle by definition).

We have the following lemma (Goldschmidt $[6(a)]$ ): 
Lemma 1.2.3. Let $R_{k} \subset J_{k}(E)$ be a kth order partial differential equation on $E$. If $R_{k+l}$ is a vector bundle, then the mth prolongation $\left(R_{k+l}\right)_{+m}$ of the equation $R_{k+l}$ is the same as the $(l+m)$ th prolongation $\left(R_{k}\right)_{+(l+m)}$ of the equation $R_{k}$.

The lemma is immediate (compare Goldschmidt loc. cit.).

Definition 1.2.7. Let $\phi: J_{k}(E) \rightarrow F$ be a bundle morphism with $R_{k}=\operatorname{ker} \phi$. We say that $\phi$, the differential operator $D=\phi \circ j_{k}: E \rightarrow F$, or $R_{k}$ is formally integrable if, for $l \geqq 0, R_{k+l}$ is a vector bundle and $\pi_{k+l}: R_{k+l+1} \rightarrow R_{k+l}$ is an epimorphism.

Formal integrability means "constancy of rank" (regularity) and "existence of formal solutions."

1.3. Resolutions for a vector bundle. We return to the Malgrange point of view (see $\$ 1.1$ ). The exterior differential $d_{X}$ with respect to the variable $x$ of $X$ gives, for each nonnegative integer $i$, a map

$$
d_{X}: \Lambda^{p} T^{*} \otimes \theta_{X \times Y} \rightarrow \Lambda^{p+1} T^{*} \otimes \mathcal{O}_{X \times Y}
$$

where (we recall) $Y$ is a copy of $X$ and $\theta_{X \times Y}$ has the $\theta$-module structure provided by $i: \mathcal{O} \rightarrow \mathcal{O}_{X \times Y}$ (see $\S 1$ ). By passage to the quotient we obtain, for each pair $p, k$ of nonnegative integers, an operator

$$
D: \Lambda^{p} T^{*} \otimes J_{k} \rightarrow \bigwedge^{p+1} T^{*} \otimes J_{k-1}
$$

which is $\mathcal{O}$-linear for the structures inherited from the right structures of $J_{k}, J_{k-1}$. Tensoring on the right by $E$, the sheaf of germs of sections of a vector bundle $E$ (more generally by a sheaf of $\mathcal{O}$-modules), we obtain the operator

$$
D: \Lambda^{p} T^{*} \otimes J_{k}(E) \rightarrow \Lambda^{p+1} T^{*} \otimes J_{k-1}(E)
$$

which is not $\mathcal{\theta}$-linear for the usual $\mathcal{\theta}$-module structures of $J_{k}(E)$, $J_{k-1}(E)$.

Let us digress for a moment to examine the local form of the operator $D$. We use the usual multi-index notation. Namely, let $\alpha=\left(\alpha_{1}, \alpha_{2}, \cdots, \alpha_{n}\right)$ be an ordered set of $n$ nonnegative integers and write

$$
\begin{aligned}
|\alpha| & =\alpha_{1}+\cdots+\alpha_{n}, \alpha !=\alpha_{1} ! \alpha_{2} ! \cdots \alpha_{n} !, \\
\alpha+1_{i} & =\left(\alpha_{1}, \cdots, \alpha_{i-1}, \alpha_{i}+1, \alpha_{i+1}, \cdots, \alpha_{n}\right) .
\end{aligned}
$$

Moreover, if $x=\left(x^{1}, \cdots, x^{n}\right), y=\left(y^{1}, \cdots, y^{n}\right)$, write

$$
(y-x)^{\alpha}=\left(y^{1}-x^{1}\right)^{\alpha_{1}}\left(y^{2}-x^{2}\right)^{\alpha_{2}} \cdots\left(y^{n}-x^{n}\right)^{\alpha_{n}} .
$$

Finally if $i=\left(i_{1}, i_{2}, \cdots, i_{p}\right)$ is a distinct set of $p$ integers between 1 and $n$, let 


$$
d x^{\wedge i}=d x^{i_{1}} \wedge d x^{i_{2}} \wedge \cdots \wedge d x^{i_{p}} .
$$

Then, locally, a section $\sigma$ of $\Lambda^{p} T^{*} \otimes J_{k}(E)$ can be written

$$
\sigma=\sum_{|\alpha| \leqslant k, i} a_{\alpha, i}(x) \frac{(y-x)^{\alpha}}{\alpha !} d x^{\wedge i} .
$$

Applying $d_{X}$ in local coordinates, we see that

$$
\text { (1.3.1) } \quad D \sigma=\sum_{|\alpha| \leqslant k-1, i ; 1 \leq j \leqq n}\left\{\frac{\partial a_{\alpha, i}}{\partial x^{j}}(x)-a_{\alpha+1, i}(x)\right\} \frac{(y-x)^{\alpha}}{\alpha !} d x^{j} \wedge d x^{\wedge i} \text {. }
$$

Thus $D$ is the difference of two operators, neither of which is defined globally, namely, the difference of the operators of actual and formal exterior derivation.

The kernel of the map

$$
d_{X}: \theta_{X \times Y} \rightarrow T^{*} \otimes \theta_{X \times Y}
$$

is the image of $\mathcal{O}$ under $j: \mathcal{O} \rightarrow \mathcal{O}_{X \times Y}$ (see $\$ 1.1$ ). By passage to the quotient, we see that the sequence

$$
0 \rightarrow \Theta \stackrel{j_{k}}{\rightarrow} J_{k} \stackrel{D}{\rightarrow} T^{*} \otimes J_{k-1}
$$

is exact. Tensoring on the right by $E$ (sheaf of sections of a vector bundle $E$ ), we obtain the exact sequence

$$
0 \rightarrow E \stackrel{j_{k}}{\rightarrow} J_{k}(E) \stackrel{D}{\rightarrow} T^{*} \otimes J_{k-1}(E) .
$$

Thus we have the sequence

$$
0 \rightarrow E \stackrel{j_{k}}{\rightarrow} J_{k}(E) \stackrel{D}{\rightarrow} T^{*} \otimes J_{k-1}(E) \stackrel{D}{\rightarrow} \cdots \stackrel{D}{\rightarrow} \Lambda^{n} T^{*} \otimes J_{k-n}(E) \rightarrow 0
$$

where, if $l<0$, we set $J_{l}(E)=0$. Since $d_{X}^{2}=0$, we obviously have $D^{2}=0$, i.e., (1.3.3) is a complex.

The following proposition is immediately verified:

Proposition 1.3.1. The differential operator (1.3.1) is uniquely characterized by the following two conditions:

(i) $D(\phi \wedge \sigma)=d \phi \wedge \pi_{k-1} \sigma+(-1)^{j} \phi \wedge D \sigma$ for each section $\sigma$ of $\Lambda^{i} T^{*} \otimes J_{k}(E)$ and differential form $\phi$ of degree $j$ (section of $\left.\Lambda^{j} T^{*}\right)$;

(ii) the sequence (1.3.2) is exact.

Condition (i) is equivalent to the assertion that the symbol of $D$ is exterior multiplication followed by the projection $\pi_{k-1}$.

We shall show that the sequence (1.3.3) is exact. Consider the diagram: 


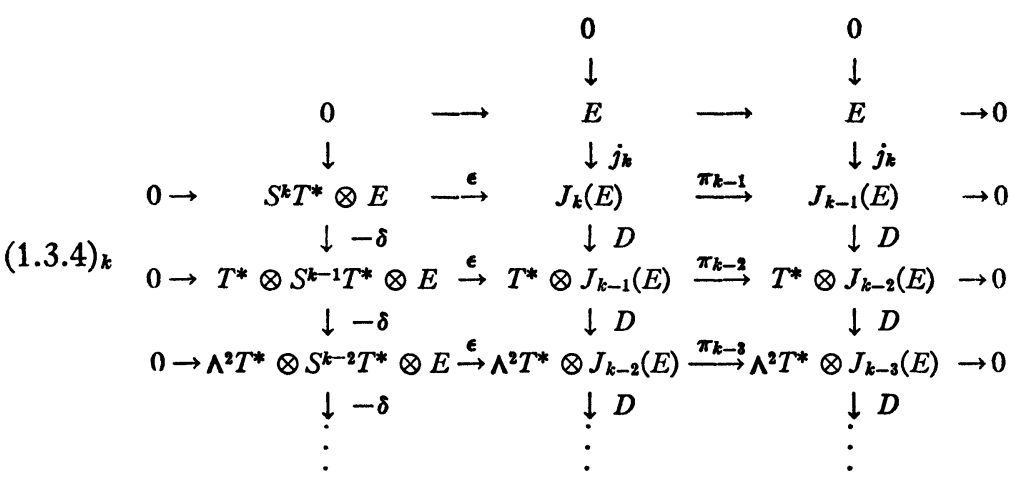

The rows are exact (compare (1.1.1)), and the maps $-\delta$ are chosen in such a way that the diagram is commutative. The minus sign preceding $\delta$ is for convenience in describing this map, as we shall now see.

In fact, $\delta$ has a natural interpretation as formal exterior differentiation and is an $\mathcal{O}$-linear map induced by morphisms

$$
\delta: \wedge^{p} T^{*} \otimes S^{m} T^{*} \rightarrow \wedge^{p+1} T^{*} \otimes S^{m-1} T^{*} .
$$

In the case $p=0, \delta$ is the composition of the natural inclusion of $S^{m} T^{*}$ into $\otimes^{m} T^{*}=T^{*} \otimes\left(\otimes^{m-1} T^{*}\right)$ and the map $T^{*} \otimes\left(\otimes^{m-1} T^{*}\right)$ $\rightarrow T^{*} \otimes S^{m-1} T^{*}$ induced by the projection $\otimes^{m-1} T^{*} \rightarrow S^{m-1} T^{*}$. The map (1.3.5), for $p>0$, sends $\omega \otimes u$ into $\delta(\omega \otimes u)=(-1)^{p} \omega \wedge \delta u$ where $\omega \in \Lambda^{p} T^{*}, u \epsilon S^{m} T^{*}$. Tensoring on the right in (1.3.5), we obtain maps

$$
\delta: \Lambda^{p} T^{*} \otimes S^{m} T^{*} \otimes E \rightarrow \Lambda^{p+1} T^{n} \otimes S^{m-1} T^{*} \otimes E
$$

sending $\omega \otimes u \otimes e$ into $\delta(\omega \otimes u) \otimes e$. Clearly $\delta^{2}=0$.

Let us examine the local form of $\delta$. An element $\eta$ of the fibre of $\Lambda^{p} T^{*} \otimes S^{m} T^{*} \otimes E$ can be written

$$
\eta=\sum_{|\alpha|=m, i} a_{\alpha, i} \frac{(y-x)^{\alpha}}{\alpha !} d x^{\wedge i}
$$

and we see immediately from (1.3.1)' (remembering to cancel the minus sign) that

$$
\delta \eta=\sum_{|\alpha|=m-1, i, j} a_{\alpha+1, i} \frac{(y-x)^{\alpha}}{\alpha !} d x^{j} \wedge d x^{\wedge i} .
$$

Thus $\delta$ is simply exterior derivation applied to polynomials, and we conclude that the left column of $(1.3 .4)_{k}$ is exact. 
It follows that the second and third columns of diagram (1.3.4) $)_{k}$ have the same cohomology and the cohomology is therefore independent of the order $k$. Since it is trivial for $k=0$, it vanishes for all $k$. Therefore, the sequence (1.3.3) is exact.

We now construct a second complex which has better properties than (1.3.3). For this purpose we consider the following portion of diagram $(1.3 .4)_{k+i+1}$ :

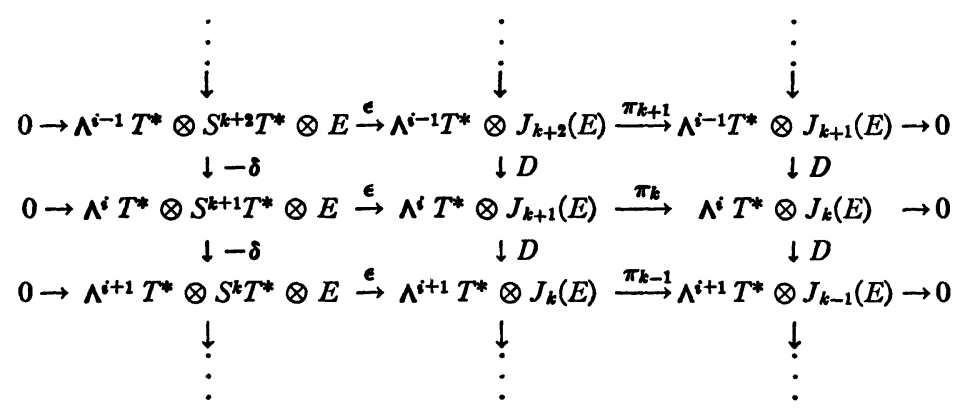

We define, for each pair $k, i$ of nonnegative integers,

$$
\begin{aligned}
C_{k}^{i+1} & =\Lambda^{i+1} T^{*} \otimes J_{k}(E) / \epsilon \delta\left(\Lambda^{i} T^{*} \otimes S^{k+1} T^{*} \otimes E\right) \\
& =\Lambda^{i+1} T^{*} \otimes J_{k}(E) / D_{\epsilon}\left(\Lambda^{i} T^{*} \otimes S^{k+1} T^{*} \otimes E\right)
\end{aligned}
$$

and we set $C_{\mathbf{k}}^{0}=J_{k}(E)$. By the diagram we have a canonical morphism

$$
\widetilde{D}: \Lambda^{i} T^{*} \otimes J_{k}(E) \rightarrow C_{k}^{i+1}
$$

which is a differential operator of order 1 . Since the kernel of $\tilde{D}$ is equal to $D\left(\bigwedge^{i-1} T^{*} \otimes J_{k+1}(E)\right)$, which contains

$$
D \in\left(\bigwedge^{i-1} T^{*} \otimes S^{k+1} T^{*} \otimes E\right),
$$

$\tilde{D}$ induces a differential operator of order 1 , namely:

$$
D^{i}: C_{k}^{i} \rightarrow C_{k}^{i+1} \text {. }
$$

The kernel of $D^{i}$ is

$$
D\left(\Lambda^{i-1} T^{*} \otimes J_{k+1}(E)\right) / D \in\left(\Lambda^{i-1} T^{*} \otimes S^{k+1} T^{*} \otimes E\right)
$$

and this is the image of $D^{i}: C_{k}^{i-1} \rightarrow C_{k}^{t}$. Therefore, we obtain, for each $k \geqq 0$, the exact sequence

$$
0 \longrightarrow E \stackrel{j_{k}}{\longrightarrow} C_{k}^{0} \stackrel{D^{0}}{\longrightarrow} C_{k}^{1} \stackrel{D^{1}}{\longrightarrow} \cdots \stackrel{D^{n-1}}{\longrightarrow} C_{k}^{n} \longrightarrow 0 .
$$

We call (1.3.3) and (1.3.8) the first and second sequences, respectively, for the vector bundle $E$. 
Finally, by passing to the projective limit and writing

$$
J_{\infty}(E)=\operatorname{proj} \lim J_{k}(E),
$$

we obtain the exact sequence

$$
0 \rightarrow E \stackrel{j_{\infty}}{\rightarrow} J_{\infty}(E) \stackrel{D}{\rightarrow} T^{*} \otimes J_{\infty}(E) \stackrel{D}{\rightarrow} \ldots \stackrel{D}{\rightarrow} \Lambda^{*} T^{*} \otimes J_{\infty}(E) \rightarrow 0 .
$$

C. Buttin [2] has constructed a formal (local) homotopy operator $K$ of degree -1 for this sequence which has meaning, in general, only in the sense of formal power series. However, in special cases (mainly in the real-analytic case) the operator $K$ can be shown to converge (see [2]). Just as the operator $D$ of the sequence (1.3.9) can be obtained by passage to the quotient from the exterior differential $d_{X}$, the operator $K$ can be obtained by passage to the quotient from the usual (local) homotopy operator $k_{X}$ for $d_{X}$ (as was pointed out by Malgrange in a conversation with $\mathrm{H}$. Goldschmidt). The operator $K$ is described in $\$ 2.1$.

1.4. The $\delta$-cohomology. We show now that the map $\delta$ of (1.3.5) induces, by restriction, a map

$$
\delta: \Lambda^{i} T^{*} \otimes g_{k+l+1} \rightarrow \Lambda^{i+1} T^{*} \otimes g_{k+l}
$$

for each pair $l, i$ of nonnegative integers. where $g_{k+m} \subset S^{k+m} T^{*} \otimes E$.

If $F$ is a vector bundle over $X$ and $\phi: J_{k}(E) \rightarrow F$ is a morphism of vector bundles, we consider the following diagram:

$$
\begin{aligned}
& J_{k+l+1}(E) \stackrel{\epsilon}{\leftarrow} S^{k+l+1} T^{*} \otimes E \stackrel{\sigma_{l+1}(\phi)}{\longrightarrow} S^{l+1} T^{*} \otimes F \stackrel{\epsilon}{\rightarrow} J_{l+1}(F)
\end{aligned}
$$

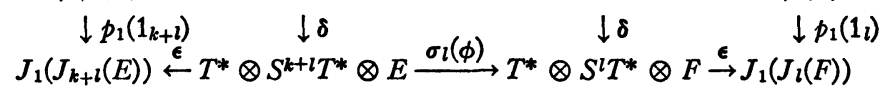

Here the first and third squares are clearly commutative, and the diagram

$$
\begin{gathered}
J_{k+l+1}(E) \stackrel{p_{l+1}(\phi)}{\longrightarrow} J_{l+1}(F) \\
\quad p_{1}\left(1_{k+1}\right) \quad p_{1}\left(1_{l}\right) \\
J_{1}\left(J_{k+l}(E)\right) \stackrel{J_{1}\left(p_{l}(\phi)\right)}{\longrightarrow} J_{1}\left(J_{l}(F)\right)
\end{gathered}
$$

is commutative. We infer that the middle square of (1.4.2) is commutative, i.e.:

$$
\begin{array}{cc}
S^{k+l+1} T^{*} \otimes E & \stackrel{\sigma_{l+1}(\phi)}{\longrightarrow} S^{l+1} T^{*} \otimes F \\
\downarrow \delta & \\
T^{*} \otimes S^{k+l} T^{*} \otimes E \stackrel{\sigma_{l}(\phi)}{\longrightarrow} T^{*} \otimes S^{l} T^{*} \otimes F
\end{array}
$$


From the commutativity of this diagram and the definition of $\delta$, we obtain the commutative diagram

$$
\begin{gathered}
\Lambda^{i} T^{*} \otimes S^{k+l+1} T^{*} \otimes E \stackrel{\sigma_{l+1}(\phi)}{\longrightarrow} \Lambda^{i} T^{*} \otimes S^{l+1} T^{*} \otimes F \\
\downarrow \delta \\
\Lambda^{i+1} T^{*} \otimes S^{k+l} T^{*} \otimes E \stackrel{\sigma_{l}(\phi)}{\longrightarrow} \Lambda^{i+1} T^{*} \otimes S^{l} T^{*} \otimes F
\end{gathered}
$$

Since $g_{k+l}$ is the kernel of $\sigma_{l}(\phi): S^{k+l} T^{*} \otimes E \rightarrow S^{l} T^{*} \otimes F$, we see that $\delta$ induces the map (1.4.1).

Definition 1.4.1. The $\delta$-cohomology of $g_{k}$ is the cohomology of the sequences

$$
\begin{aligned}
0 & \rightarrow g_{m} \stackrel{\delta}{\rightarrow} T^{*} \otimes g_{m-1} \stackrel{\delta}{\rightarrow} \Lambda^{2} T^{*} \otimes g_{m-2} \stackrel{\delta}{\rightarrow} \cdots \\
& \stackrel{\delta}{\rightarrow} \Lambda^{m-k} T^{*} \otimes g_{k} \stackrel{\delta}{\rightarrow} \Lambda^{m-k+1} T^{*} \otimes S^{k-1} T^{*} \otimes E
\end{aligned}
$$

where $m \geqq k$. We denote by $H^{m-i, i}=H^{m-i, i}\left(g_{k}\right)$ the cohomology of the sequence (1.4.4) at $\Lambda^{i} T^{*} \otimes g_{m-i}$. We say that $g_{k}$ is involutive if the sequences (1.4.4) are exact. We say that $g_{k}$ is $q$-acyclic if $H^{m, i}=0$ for $m \geqq k, 0 \leqq i \leqq q$.

We remark that, for each $l \geqq 0$, the sequence

$$
0 \rightarrow g_{k+l+1} \stackrel{\delta}{\rightarrow} T^{*} \otimes g_{k+l} \stackrel{\delta}{\rightarrow} \Lambda^{2} T^{*} \otimes g_{k+l-1}
$$

is easily seen to be exact.

ThEOREM 1.4.1 ( $\delta$-POINCARÉ LemMA). There exists an integer $\mu \geqq k$, depending only on $n$ (dimension of $X$ ), $k$ (order of the differential operator) and (fibre) dimension of $E$ (sup $\operatorname{dim} E$ if $X$ is not connected), such that $H^{m, i}=0$ for all $m \geqq \mu$ and $i \geqq 0$.

Remark. Suppose that $\operatorname{dim} E_{x} \leqq d$ for $x \in X$. Then the integer $\mu=\mu(n, d, k)$ can be calculated from the following relations:

$$
\mu(0, d, 1)=0 ;
$$

(ii) $\mu(n, d, 1)=d \cdot\left(\begin{array}{l}a+n \\ n-1\end{array}\right)+a+1$, where $a=\mu(n-1, d, 1)$;

(iii) $\quad \mu(n, d, k)=\mu(n, b, 1) \quad$ where $\quad b=\sum_{\nu=0}^{k}\left(\begin{array}{c}\nu+n-1 \\ n-1\end{array}\right) \cdot d$.

For a proof see Sweeney $[23(b)]$ (see also [21(c)] and [6(c)]).

1.5. Criterion for formal integrability and reduction to a formally integrable equation. H. Goldschmidt, in his paper [6(a)], has given a 
criterion, involving only a finite number of prolongations, in order that a partial differential equation be formally integrable, and it is based on the following theorem.

THEOREM 1.5.1. Let $\phi: J_{k}(E) \rightarrow F$ be a linear map with $R_{k}=\operatorname{ker} \phi$. Then it is formally integrable if

(i) $R_{k+1}$ is a vector bundle;

(ii) $\pi_{k}: R_{k+1} \rightarrow R_{k}$ is surjective;

(iii) $g_{k}$ is 2-acyclic.

We have also (Goldschmidt, loc. cit.):

Proposition 1.5.1. If, for some $l \geqq 1, R_{k+l}$ is a vector bundle and $\pi_{k+l-1}: R_{k+l} \rightarrow R_{k+l-1}$ is surjective, then $R_{k+l-1}$ is a vector bundle.

From Theorem 1.5.1, Proposition 1.5.1 and Theorem 1.4 .1 ( $\delta$ Poincaré lemma), we obtain (Goldschmidt, loc. cit.):

Theorem 1.5.2 (CRIterion for Formal Integrability). Let $\phi: J_{k}(E) \rightarrow F$ be a linear map, and suppose that $\operatorname{dim} E_{x} \leqq d$ for all $x \in X$. Then there exists an integer $\mu, \mu \geqq k$, where $\mu$ depends only on $n$ (dimension of $X), k$ and $d$, such that if, for some $m \geqq \mu, R_{m+1}$ is a vector bundle and $\pi_{k+l}: R_{k+l+1} \rightarrow R_{k+l}$ is surjective for $0 \leqq l \leqq m-k$, then $\phi$ is formally integrable.

If $R_{m}$ is a sub-bundle of $J_{m}(E)$ we shall denote, in the following theorem, by $\left(R_{m}\right)_{+l}$ the $l$ th prolongation of $R_{m}$, i.e. (compare Definition 1.2.5), $\left(R_{m}\right)_{+l}=J_{l}\left(R_{m}\right) \cap J_{m+l}(E)$. In his fundamental paper [14(a)] M. Kuranishi proved the following theorem:

Theorem 1.5.3 (Cartan-Kuranishi Prolongation Theorem). For each $m \geqq k$, suppose that a sub-bundle $R_{m}$ of $J_{m}(E)$ is given and that $R_{m+1} \subset\left(R_{m}\right)_{+1}, R_{m+1} \rightarrow R_{m}$ surjective. Then there exists an integer $m_{0}$ such that, for all $l \geqq 0, R_{m_{0}+l}=\left(R_{m_{0}}\right)_{+l}$.

In the remainder of this section we summarize the main results proved by Goldschmidt in his paper [6(c), (d)].

Definition 1.5.1. Let $E, F$ be vector bundles over $X$. We say that a differential operator $\phi: J_{k}(E) \rightarrow F$ is regular, if for each $l \geqq 0$, the morphism $p_{l}(\phi): J_{k+l}(E) \rightarrow J_{l}(F)$ has constant rank or, equivalently, the kernel $R_{k+l}$ of $p_{l}(\phi)$ (the $l$ th prolongation of $R_{k}$ ) is a vector bundle. Similarly, we say that $R_{k}=\operatorname{ker} \phi$ is regular if $R_{k}$ and all its prolongations are vector bundles.

We suppose in the remainder of this section that $X$ is connected.

Theorem 1.5.4 (Cartan-Goldschmidt Prolongation Theorem). Let $R_{k} \subset J_{k}(E)$ be a regular partial differential equation, and suppose 
that the maps $\pi_{k+l}: R_{k+l+m} \rightarrow R_{k+l}$ have constant rank for all $l, m \geqq 0$. Then there exist integers $l_{0} \geqq 0, m_{0} \geqq k$, such that the equation $R_{m_{0}}^{\left(l_{0}\right)}$ $=\pi_{m_{0}}\left(R_{m_{0}+l_{0}}\right)$ of order $m_{0}$ on $E$ is a formally integrable, "involutive equation" which has the same formal solutions as $R_{k}$ and whose rth prolongation is $R_{m_{0}+r}^{\left(l_{0}\right)}$.

Let us say that a differential operator $D: E \rightarrow F$ of order $k$ is "sufficiently regular" if it is regular and its equation satisfies the hypothesis of Theorem 1.5.4. On the basis of the prolongation theorem (Theorem 1.5.4) Goldschmidt shows that, if $D: E \rightarrow F$ is a sufficiently regular operator, there exist a vector bundle $F_{1}$ and differential operator $P: F \rightarrow F_{1}$, which can be constructed in finitely many steps, such that $D_{1}=P \circ D: E \rightarrow F_{1}$ is formally integrable and the solutions of the equations $D e=f$ and $D_{1} e=P f$ are the same if $f$ satisfies compatibility conditions. Moreover, if $f$ satisfies the necessary compatibility conditions so does $P f$ and, if $f_{1} \in F_{1}$ satisfies the necessary compatibility conditions for $D_{1}$, then there exists $f \in F$ satisfying $P f=f_{1}$ and the necessary compatibility conditions for $D$. In short, a sufficiently regular differential operator can be replaced by a formally integrable operator which is equivalent to it. More precisely, the following two theorems hold.

ThEOREM 1.5.5. Let $D: E \rightarrow F$ be a regular differential operator of order $k$ with equation $R_{k} \subset J_{k}(E)$. Then

(i) There exist a bundle $G$ and differential operator $D^{\prime}: F \rightarrow G$ of order $l$ such that $p\left(D^{\prime}\right): J_{l}(F) \rightarrow G$ is an epimorphism and, for each $r \geqq 0$, the sequence

$$
0 \longrightarrow R_{k+l+r} \longrightarrow J_{k+l+r}(E) \stackrel{p_{l+r}(D)}{\longrightarrow} J_{l+r}(F) \stackrel{p_{r}\left(D^{\prime}\right)}{\longrightarrow} J_{r}(G)
$$

is exact.

(ii) Let $D^{\prime \prime}: F \rightarrow H$ be any differential operator of order $m$ such that, for each $r \geqq 0$, the sequence

$$
J_{k+m+r}(E) \stackrel{p_{m+r}(D)}{\longrightarrow} J_{m+r}(F) \stackrel{p_{r}\left(D^{\prime \prime}\right)}{\longrightarrow} J_{r}(H)
$$

is exact. Then $m \geqq l$ and there is a differential operator $\bar{D}: G \rightarrow H$ satisfying $D^{\prime \prime}=\bar{D} \circ D^{\prime}$.

(iii) The operator $D^{\prime}$ is essentially unique in the following sense. Let $G_{1}$ be $a$ vector bundle and $D_{1}^{\prime}: F \rightarrow G_{1}$ any differential operator of the same order as $D^{\prime}$ and satisfying the same conditions. Then there exists an isomorphism $\psi: G \rightarrow G_{1}$ of vector bundies such that $D_{1}^{\prime}=\psi \circ D^{\prime}$. 
Definition 1.5.2. We say that $f \in F$ satisfies the compatibility condition for the inhomogeneous equation $D e=f$ if $D^{\prime} f=0$ where $D^{\prime}$ is the operator of Theorem 1.5.5.

We remark that the compatibility condition is independent of the choice of $D^{\prime}$ satisfying the conditions (i) of Theorem 1.5.5.

THEOREM 1.5.6. Let $D: E \rightarrow F$ be a regular differential operator of order $k$ with equation $R_{k} \subset J_{k}(E)$, and suppose that the maps $\pi_{k+r}$ : $R_{k+r+s} \rightarrow R_{k+r}$ have constant rank for all $r, s \geqq 0$. Then there exist a bundle $F_{1}$ and differential operator $P: F \rightarrow F_{1}$ of order $l_{0}+m_{0}$, which can be defined in finitely many steps, such that the following assertions hold:

(i) The operator $D_{1}=P \circ D: E \rightarrow F_{1}$ has order $k+m_{0}$, is formally integrable and has the same solutions and formal solutions as $D$.

(ii) Let $D^{\prime}: F \rightarrow G$ be a differential operator of order $l$ satisfying the conditions of (i), Theorem 1.5.5, and let $D_{1}^{\prime}: F_{1} \rightarrow G_{1}$ be a differential operator of order s such that, for each $r \geqq 0$, the sequence

$$
J_{k+m_{0}+s+r}(E) \stackrel{p_{s+r}\left(D_{1}\right)}{\longrightarrow} J_{s+r}\left(F_{1}\right) \stackrel{p_{r}\left(D_{1}^{\prime}\right)}{\longrightarrow} J_{r}\left(G_{1}\right)
$$

is exact. Then there exist an integer $m$ and differential operator $Q: G$ $\rightarrow J_{m}\left(G_{1}\right)$ satisfying the following conditions:

(a) The diagram commutes:

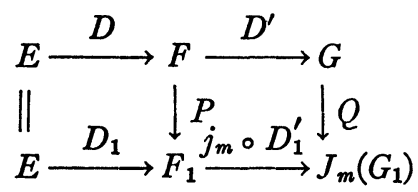

Hence, if $f \in F$ satisfies $D^{\prime} f=0$, then $D_{1}^{\prime}(P f)=0$.

(b) For $f \in F$, satisfying the compatibility conditions, the solutions $e \in E$ of the inhomogeneous differential equation $D e=f$ coincide with the solutions of the equation $D_{1} e=P f$. Moreover, $P$ induces an isomorphism from the cohomology of the complex

$$
E \stackrel{D}{\longrightarrow} F \stackrel{D^{\prime}}{\longrightarrow} G
$$

to the cohomology of the complex

$$
E \stackrel{D_{1}}{\rightarrow} F_{1} \stackrel{D_{1}^{\prime}}{\longrightarrow} G_{1} .
$$

Hence, if $f_{1} \in F_{1}$ satisfies $D_{1}^{\prime} f_{1}=0$, there exists $f \in F$ satisfying $P f=f_{1}$ and $D^{\prime} f=0$. 
Finally, we list the following theorem:

THEOREM 1.5.7. Let $\phi: J_{k}(E) \rightarrow F$ be a regular differential operator of order $k$ and set $D_{0}=\phi \circ j_{k}$. Then there exists a complex

$$
\begin{aligned}
& 0 \longrightarrow \Theta \longrightarrow E \stackrel{D_{0}}{\longrightarrow} G_{0} \stackrel{D_{1}}{\longrightarrow} G_{1} \stackrel{D_{2}}{\longrightarrow} G_{2} \stackrel{D_{3}}{\longrightarrow} \cdots \\
& \stackrel{D_{r}}{\longrightarrow} G_{r} \stackrel{D_{r+1}}{\longrightarrow} \ldots
\end{aligned}
$$

where $G_{r}$ is a vector bundle and $G_{0}=F$, and $D_{r}=\phi_{r} \circ j_{l_{r}}: G_{r \rightarrow 1} \rightarrow G_{r}$ is a differential operator of order $l_{r}$, which is exact at $E(\Theta$ is the sheaf of solutions of the homogeneous equation) and formally exact in the sense that the sequences

$$
\begin{aligned}
& 0 \longrightarrow R_{k+m} \longrightarrow J_{k+m}(E) \stackrel{p_{m}\left(D_{0}\right)}{\longrightarrow} J_{m}\left(G_{0}\right) \stackrel{p_{m-l_{1}}\left(D_{1}\right)}{\longrightarrow} J_{m-l_{1}}\left(G_{1}\right) \longrightarrow \cdots \\
& \cdots \longrightarrow J_{m-l_{1}-\cdots-l_{r}}\left(G_{r}\right) \longrightarrow \cdots
\end{aligned}
$$

are exact at $R_{k+m}$ and $J_{k+m}(E)$ for $m \geqq 0$, at $J_{m}\left(G_{0}\right)$ for $m \geqq l_{1}$, and at $J_{m-l_{1}-\cdots-l_{r}}\left(G_{r}\right)$ for $m \geqq l_{1}+\cdots+l_{r+1}, r \geqq 1$.

In its original form (with stronger assumptions) this theorem is due to Kuranishi [14(b)]; in its present form it is due to Goldschmidt [6(a)] (see also Quillen [19]).

1.6. Complexes (sequences) attached to a formally integrable differential operator. Let $D=\phi \circ j_{k}: E \rightarrow F$ be a differential operator corresponding to the bundle morphism $\phi: J_{k}(E) \rightarrow F$, which for simplicity we assume to be formally integrable, let $R_{k} \subset J_{k}(E)$ be its equation, $R_{k+l}$ its $l$ th prolongation and $g_{k+l}$ the kernel of the map $\pi_{k+l-1}: R_{k+l}$ $\rightarrow R_{k+l-1}$ (as in $\S 1.2$ ).

The operator (1.3.1) induces a differential operator

$$
D: \Lambda^{i} T^{*} \otimes R_{k+l+1} \rightarrow \Lambda^{i+1} T^{*} \otimes R_{k+l}
$$

for each pair $i, l$ of nonnegative integers. In fact, we have only to verify that the following diagram commutes:

$$
\begin{array}{ccc}
\Lambda^{i} T^{*} \otimes & J_{k+l+1}(E) \stackrel{p_{l+1}(\phi)}{\longrightarrow} \bigwedge^{i} T^{*} \otimes J_{l+1}(F) \\
& \downarrow D & \downarrow D \\
\Lambda^{i+1} T^{*} & \otimes J_{k+l}(E) \stackrel{p_{l}(\phi)}{\longrightarrow} \Lambda^{i+1} T^{*} \otimes J_{l}(F)
\end{array}
$$

To see that it does, introduce the difference $p_{l}(\phi) \circ D-D \circ p_{l+1}(\phi)$ and use Proposition 1.3.1.

Let $\Theta$ denote the sheaf of germs of solutions of $R_{k}$ (solutions of the homogeneous equation), i.e. (see Definition 1.2.5) 


$$
\Theta=\left\{e \mid e \in E, j_{k}(e) \in R_{k}\right\} .
$$

Then, for $m \geqq k+n$, we have the following commutative diagram (compare $\left.(1.3 .4)_{k}\right)$ :

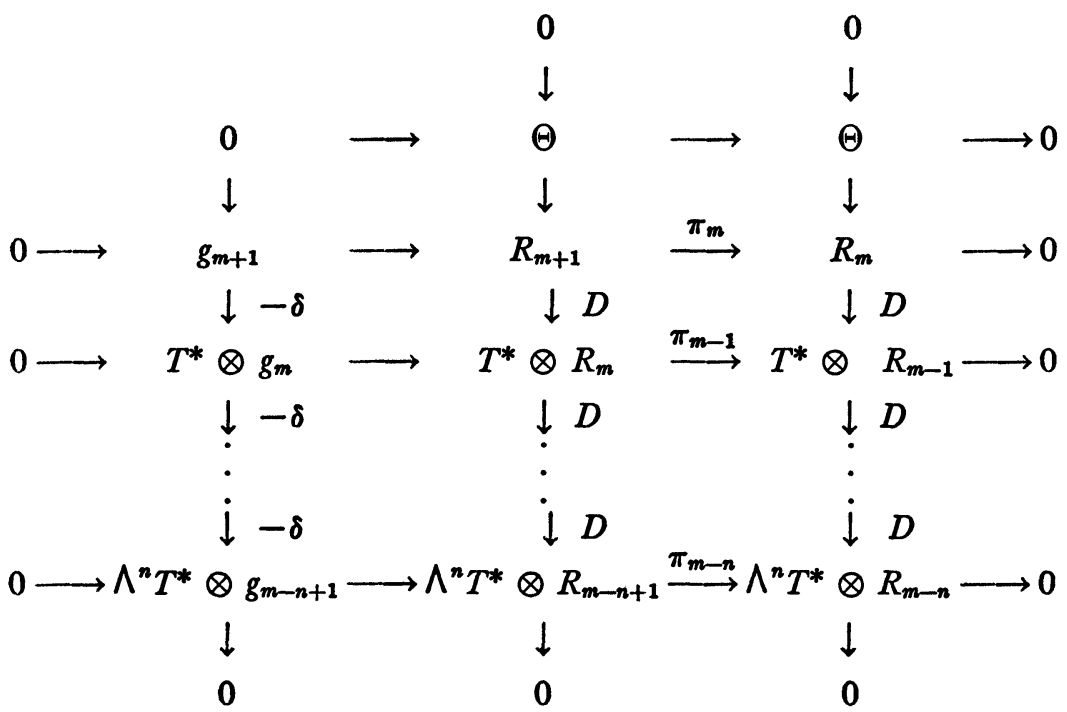

The rows are exact since $R_{k}$ is formally integrable, and the diagram is clearly exact at $R_{m+1}$ and $R_{m}$ (see Proposition 1.3.1, (ii)). Now suppose that $m \geqq \mu+n-1$, where $\mu$ is the integer of Theorem 1.4.1. Then (by Theorem 1.4.1) the left column of this diagram is exact, and it follows that the cohomology of the sequence

$$
0 \rightarrow \Theta \stackrel{j_{m}}{\rightarrow} R_{m} \stackrel{D}{\rightarrow} T^{*} \otimes R_{m-1} \stackrel{D}{\rightarrow} \cdots \stackrel{D}{\rightarrow} \Lambda^{n} T^{*} \otimes R_{m-n} \rightarrow 0
$$

is independent of $m$ if $m$ is large enough. We call (1.6.2) the first sequence (complex) attached to the differential operator, and we say that $m$ is in the stable range if it is large enough for the cohomology of (1.6.2) to be stable.

Next, we shall define a second sequence (complex) attached to the differential operator which bears the same relationship to (1.3.8) as (1.6.2) bears to (1.3.3). We set $C^{0}=C_{m}^{0}=R_{m}$ and define

$$
C^{i}=C_{m}^{i}=\left(\bigwedge^{i} T^{*} \otimes R_{m}\right) / \delta\left(\bigwedge^{i-1} T^{*} \otimes g_{m+1}\right)
$$

for $i \geqq 1$.

Lemмa 1.6.1. Suppose that $m \geqq \mu$ (the integer of Theorem 1.4.1). Then the $C^{i}, 0 \leqq i \leqq n$, are vector bundles. 
The following proof (due to Goldschmidt [6(a)]) depends on the following standard (and trivial) lemma:

Lemma 1.6.2. Let

$$
0 \longrightarrow V_{0} \stackrel{T_{0}}{\longrightarrow} V_{1} \stackrel{T_{1}}{\longrightarrow} V_{2} \stackrel{T_{2}}{\longrightarrow} \ldots \stackrel{T_{r-1}}{\longrightarrow} V_{r} \longrightarrow 0
$$

be a complex (i.e., $T_{i+1} \circ T_{i}=0$ ) where each $V_{i}$ is a finite-dimensional vector space and the maps $T_{i}: V_{i} \rightarrow V_{i+1}$ are linear. Denote by $H^{i}$ the cohomology at $V_{i}$, i.e.,

$$
H^{i}=\operatorname{ker} T_{i} / \operatorname{im} T_{i-1}, \quad i=0,1, \cdots, r .
$$

Then (Euler formula)

$$
\sum_{i=0}^{r}(-1)^{i} \operatorname{dim} B^{i}=\sum_{i=0}^{r}(-1)^{i} \operatorname{dim} V_{i} .
$$

Proof of Lemma 1.6.1. We remark first that $C^{0}=R_{m}$ is a vector bundle, and $C^{1}$ is a vector bundle because $g_{m+1}$ is a vector bundle and the map

$$
\delta: g_{m+1} \rightarrow T^{*} \otimes g_{m}
$$

is injective. Suppose now that $m \geqq \mu$ (the integer of Theorem 1.4.1). Then, for each $i \geqq 1$, the sequence

$$
0 \rightarrow K \rightarrow \Lambda^{i-1} T^{*} \otimes g_{m+2} \stackrel{\delta}{\rightarrow} \Lambda^{i} T^{*} \otimes g_{m+1} \stackrel{\delta}{\rightarrow} \Lambda^{i+1} T^{*} \otimes R_{m} \stackrel{p}{\rightarrow} C^{i+1} \rightarrow 0
$$

is exact, where $K$ is the kernel of $\delta: \Lambda^{i-1} T^{*} \otimes g_{m+2} \rightarrow \Lambda^{i} T^{*} \otimes g_{m+1}$ and $p: \Lambda^{i+1} T^{*} \otimes R_{m} \rightarrow C^{i+1}$ is the natural map. Since the sequence is exact, the cohomology vanishes and we have by Lemma 1.6.2, for each $x \in X$,

$$
\begin{aligned}
\operatorname{dim} K_{x}+\operatorname{dim} C_{x}^{i+1}=\operatorname{dim}\left(\Lambda^{i-1} T^{*} \otimes g_{m+2}\right)_{x} & -\operatorname{dim}\left(\Lambda^{i} T^{*} \otimes g_{m}\right)_{x} \\
& +\operatorname{dim}\left(\Lambda^{i+1} T^{*} \otimes R_{m}\right)_{x} .
\end{aligned}
$$

On each connected component of $X$, the right side of this equation is constant (independent of $x \in X$ ) since only the dimensions of the fibres of vector bundles occur there, and hence the sum on the left is constant. Since $K_{x}$ is the kernel, $C_{x}^{t+1}$ the cokernel, of morphisms of vector bundles, $\operatorname{dim} K_{x}$ and $\operatorname{dim} C_{x}^{+1}$ are upper semicontinuous functions of $x$ and it follows that, on each component of $X, \operatorname{dim} K_{x}$ and $\operatorname{dim} C_{x}^{i+1}$ are constant. Therefore, in particular, $C^{i+1}$ is a vector bundle.

In a manner similar to that used in $\$ 1.3$, we can define operators 


$$
D^{i}: C^{i} \rightarrow C^{i+1}
$$

In fact, consider the diagram

$$
\begin{aligned}
& 0 \rightarrow \Lambda^{i} T^{*} \otimes g_{m+1} \rightarrow \Lambda^{i} T^{*} \otimes R_{m+1} \stackrel{\pi_{m}}{\rightarrow} \Lambda^{i} T^{*} \otimes R_{m} \rightarrow 0 \\
& \downarrow-\delta \quad \downarrow D \quad \downarrow \tilde{D} \\
& 0 \rightarrow \delta\left(\Lambda^{i} T^{*} \otimes g_{m+1}\right) \rightarrow \Lambda^{i+1} T^{*} \otimes R_{m} \stackrel{p}{\longrightarrow} C^{i+1} \quad \rightarrow 0
\end{aligned}
$$

where the rows are exact and $\widetilde{D}$ is induced by $D$. It is easy to see that $\tilde{D}$ factors through $C^{i}$, i.e., there is an operator (1.6.4) such that $\tilde{D}=\tilde{D}^{i} \circ p$ and $D^{i+1} \circ D^{i}=0$. Thus, we obtain a second sequence (complex) attached to the differential operator.

However, in order to determine more precisely the properties of the second sequence, we shall follow the natural procedure of constructing this sequence introduced by Goldschmidt [6(a)] and shall obtain as by-product the following theorem (Goldschmidt, loc. cit.):

THEOREM 1.6.1. Let $D: E \rightarrow F$ be a formally integrable differential operator of order $k$ with equation $R_{k} \subset J_{k}(E)$. Let $m$ be a fixed integer, $m \geqq \mu$ (the integer of Theorem 1.4.1) and set

$$
C^{0}=C_{m}^{0}=R_{m} .
$$

Then there exists a complex (second sequence attached to the differential operator)

$$
0 \longrightarrow \Theta \stackrel{j_{m}}{\longrightarrow} C^{0} \stackrel{D^{0}}{\longrightarrow} C^{1} \stackrel{D^{1}}{\longrightarrow} \ldots \stackrel{D^{n-1}}{\longrightarrow} C^{n} \longrightarrow 0
$$

which is uniquely determined up to isomorphisms by the following properties:

(i) For each $i, 0 \leqq i \leqq n-1$, the map $D^{i}: C^{i} \rightarrow C^{i+1}$ is a first-order differential operator induced by a morphism $\rho^{i}: J_{1}\left(C^{i}\right) \rightarrow C^{i+1}$ of vector bundles, whose symbol is a surjective morphism $\sigma\left(D^{i}\right): T^{*} \otimes C^{i} \rightarrow C^{i+1}$ induced by the multiplication map $T^{*} \otimes \Lambda^{i} T^{*} \rightarrow \Lambda^{i+1} T^{*}$.

(ii) The sequence

$$
0 \rightarrow \Theta \stackrel{j_{m}}{\rightarrow} C^{0} \stackrel{D^{0}}{\rightarrow} C^{1}
$$

is exact.

(iii) The sequence (1.6.6) is formally exact in the sense that the sequences

$$
0 \longrightarrow R_{m+l} \longrightarrow J_{l}\left(C^{0}\right) \stackrel{p_{l-1}\left(\rho^{0}\right)}{\longrightarrow} J_{l_{1}}\left(C^{1}\right) \stackrel{p_{l-2}\left(\rho^{1}\right)}{\longrightarrow} \ldots
$$


are exact at $R_{m+l}$ for $l \geqq 1$, and at $J_{l-i}\left(C^{i}\right)$ for $l \geqq i+1,0 \leqq i \leqq r-1$.

We can identify the $C^{i}$ of the theorem with the $C^{i}$ defined by (1.6.3).

Proof of Theorem 1.6.1 (Goldschmidt [6(a)]). We suppose that $m$ is a fixed integer, $m \geqq \mu$ (the integer of Theorem 1.4.1), and that the $C^{i}=C_{m}^{i}$ are defined by (1.6.3) for $i \geqq 1, C^{0}=R_{m}$.

We define the operators $D^{i}$ successively, and we begin with $D^{0}: C^{0} \rightarrow C^{1}$. We have the exact commutative diagram

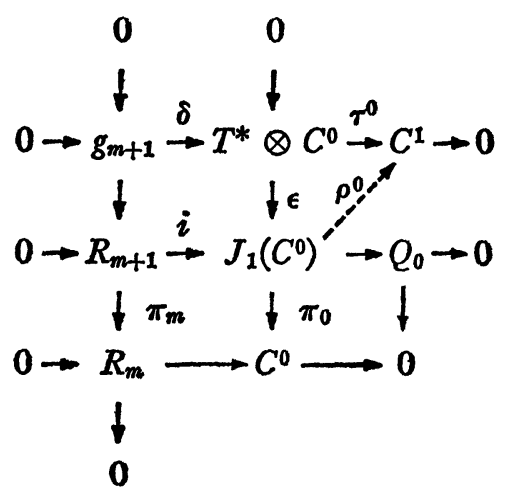

Here $Q_{0}$ is the cokernel of $i$, the inclusion of $R_{m+1}$ in $J_{1}\left(C^{0}\right)=J_{1}\left(R_{m}\right)$, and $\tau^{0}$ is the natural projection. The diagram induces an isomorphism between $Q_{0}$ and $C^{1}$ and therefore it also induces a morphism $\rho^{0}: J_{1}\left(C^{0}\right) \rightarrow C^{1}$ such that the sequence

$$
0 \rightarrow R_{m+1} \stackrel{i}{\rightarrow} J_{1}\left(C^{0}\right) \stackrel{\rho^{0}}{\rightarrow} C^{1} \rightarrow 0
$$

is exact and $\tau^{0}=\rho^{0} \circ \epsilon$. The uniqueness of $\rho^{0}$ follows from the exactness of (1.6.8) and the fact that $\tau^{0}$ is an epimorphism. We have the exact commutative diagram

$$
\begin{array}{ccc}
0 & 0 \\
& \downarrow & \\
J_{l}\left(R_{m}\right) & \stackrel{p_{l-1}\left(\rho^{0}\right)}{\longrightarrow} J_{l-1}\left(C^{1}\right) \\
J_{l-1}\left(R_{m+1}\right) \stackrel{J_{l-1}(i)}{\longrightarrow} J_{l-1}\left(J_{1}\left(R_{m}\right)\right) \stackrel{J_{l-1}\left(\rho^{0}\right)}{\longrightarrow} J_{l-1}\left(C^{1}\right)
\end{array}
$$

The lower row is obtained by applying the functor $J_{l-1}$ to the sequence (1.6.8), and the square commutes by Lemma 1.2.2. The kernel of $p_{l-1}\left(\rho^{0}\right)$ therefore is 


$$
K=J_{l}\left(R_{m}\right) \cap J_{l-1}(i)\left(J_{l-1}\left(R_{m+1}\right)\right)
$$

Since $R_{m+1}=J_{1}\left(R_{m}\right) \cap J_{m+1}(E)$ (by definition), we have

$$
\begin{aligned}
K & =J_{l}\left(R_{m}\right) \cap J_{l-1}(i)\left(J_{l-1}\left(J_{1}\left(R_{m}\right) \cap J_{m+1}(E)\right)\right) \\
& =J_{l}\left(R_{m}\right) \cap J_{l-1}\left(J_{1}\left(R_{m}\right)\right) \cap J_{l-1}\left(J_{m+1}(E)\right) \\
& =J_{l}\left(R_{m}\right) \cap J_{l-1}\left(J_{m+1}(E)\right)
\end{aligned}
$$

since $J_{l}\left(R_{m}\right) \subset J_{l-1}\left(J_{1}\left(R_{m}\right)\right)$. Since

$$
J_{l}\left(R_{m}\right) \cap J_{l-1}\left(J_{m+1}(E)\right) \subset J_{l}\left(J_{m}(E)\right) \cap J_{l-1}\left(J_{m+1}(E)\right)=J_{l+m}(E)
$$

by Lemma 1.2.1, we have

$$
J_{l}\left(R_{m}\right) \cap J_{l-1}\left(J_{m+1}(E)\right)=J_{l}\left(R_{m}\right) \cap J_{l+m}(E)=R_{l+m},
$$

i.e.,

$$
K=R_{l+m}
$$

Therefore, for $l \geqq 1$, the sequences

$$
0 \longrightarrow R_{m+l} \longrightarrow J_{l}\left(C^{0}\right) \stackrel{p_{l-1}\left(\rho^{0}\right)}{\longrightarrow} J_{l-1}\left(C^{1}\right)
$$

are exact, and the $l$ th prolongation of the first-order equation $R_{m+1} \subset J_{1}\left(C^{0}\right)$ on $C^{0}=R_{m}$ is $R_{m+l+1} \subset J_{l+1}\left(C^{0}\right)$.

Let $D^{0}: C^{0} \rightarrow C^{1}$ be the first-order differential operator $\rho^{0} \circ j_{1}$, whose symbol $\sigma\left(D^{0}\right)$ is equal to $\tau^{0}$. Then the sequence

$$
0 \rightarrow \Theta \stackrel{j_{m}}{\rightarrow} C^{0} \stackrel{D^{0}}{\rightarrow} C^{1}
$$

is exact. We omit a proof since it is straightforward.

Next, we construct $D^{1}: C^{1} \rightarrow C^{2}$. Consider the following diagram:

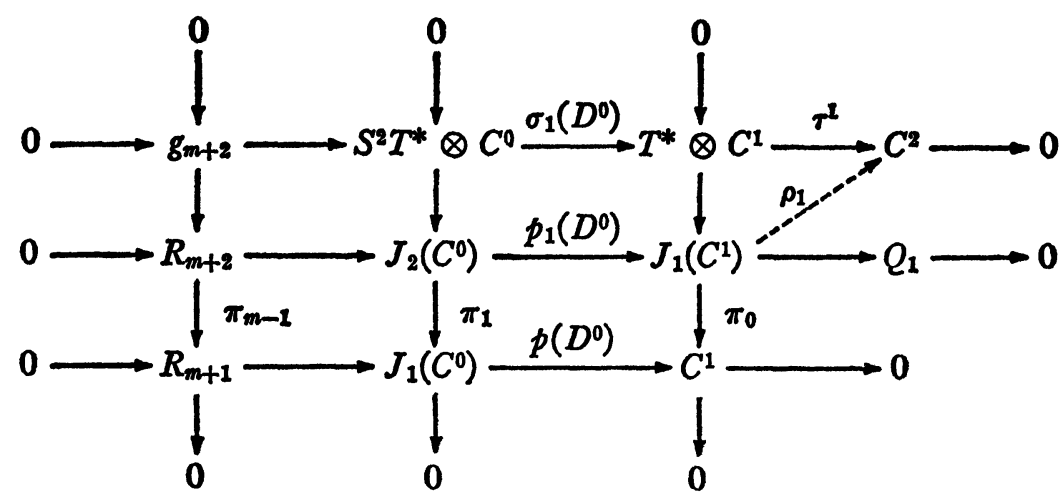


Here $\tau^{1}: T^{*} \otimes C^{1} \rightarrow C^{2}$ is the epimorphism induced by the multiplication map $T^{*} \otimes \Lambda^{i} T^{*} \rightarrow \Lambda^{i+1} T^{*}$, and $Q_{1}$ is the cokernel of $p_{1}\left(D^{0}\right): J_{2}\left(C^{0}\right)$ $\rightarrow J_{1}\left(C^{1}\right)$. The columns are exact, and the second and third rows are exact by (1.6.8), (1.6.10) and the definition of $Q_{1}$. Suppose that the top row is exact. Then the diagram induces an isomorphism between $Q_{1}$ and $C^{2}$, and therefore it also induces a morphism $\rho^{1}: J_{1}\left(C^{1}\right) \rightarrow C^{2}$, whose symbol is $\tau^{1}$, such that the sequence

$$
0 \rightarrow R_{m+2} \rightarrow J_{2}\left(C^{0}\right) \stackrel{p_{1}\left(D^{0}\right)}{\longrightarrow} J_{1}\left(C^{1}\right) \stackrel{\rho^{1}}{\rightarrow} C^{2} \rightarrow 0
$$

is exact. The uniqueness of $\rho^{1}$ follows from the exactness of (1.6.11) and the fact that $\tau^{1}$ is an epimorphism. We then let $D^{1}: C^{1} \rightarrow C^{2}$ be the first-order operator $\rho^{1} \circ j_{1}$, whose symbol $\sigma\left(D^{1}\right)$ is equal to $\tau^{1}$.

We shall now show that the top row of the preceding diagram is exact. First, consider the commutative diagram

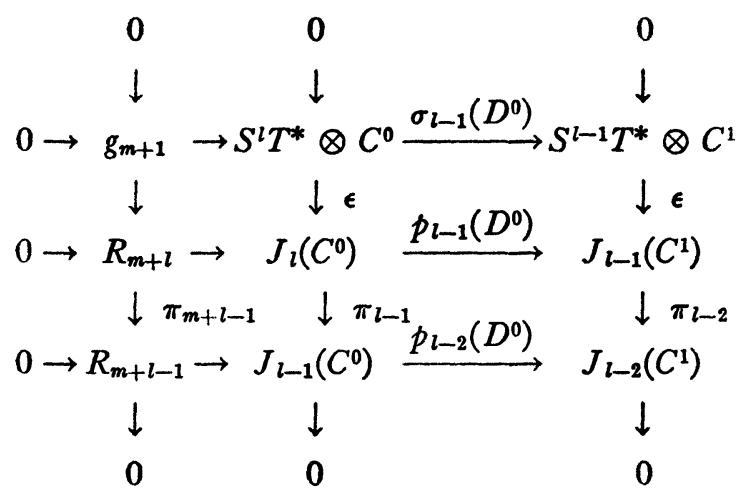

The map $\sigma_{l-1}\left(D^{0}\right)$ is given by

$$
\sigma_{l-1}\left(D^{0}\right)=\left(1 \otimes \sigma\left(D^{0}\right)\right) \circ \delta
$$

where

$$
\delta: S^{l+1} T^{*} \otimes C^{0} \rightarrow S^{l} T^{*} \otimes T^{*} \otimes C^{0} .
$$

The columns are exact, and the second and third rows are exact by (1.6.10), and it follows that the first row is exact also, i.e., for $l \geqq 1$, the sequence

$$
0 \rightarrow g_{m+l} \rightarrow S^{l} T^{*} \otimes C^{0} \stackrel{\sigma_{l-1}\left(D^{0}\right)}{\longrightarrow} S^{l-1} T^{*} \otimes C^{1}
$$

is exact.

Next, we have the exact commutative diagram 


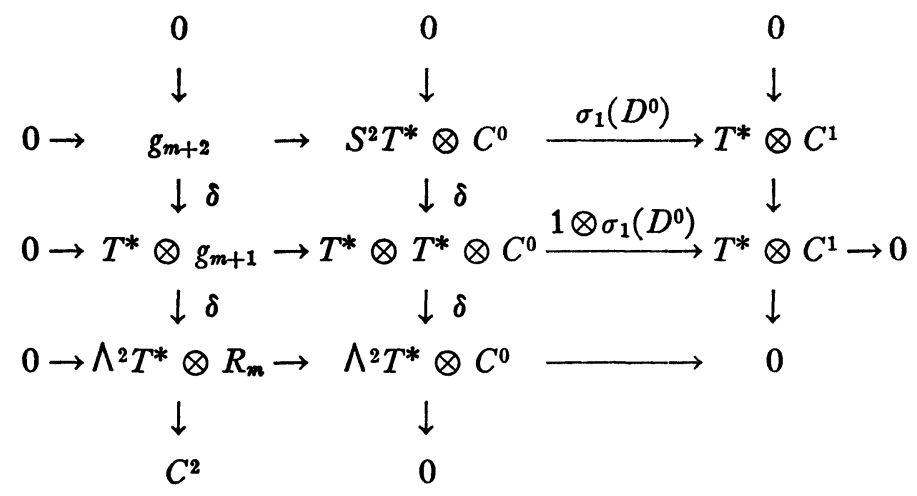

The first row is exact by (1.6.12) with $l=2$, and the second row is exact since the symbol of $D^{0}$ is surjective. The diagram defines a map $\tau^{1}: T^{*} \otimes C^{1} \rightarrow C^{2}$ such that the sequence

$$
0 \rightarrow g_{m+2} \rightarrow S^{2} T^{*} \otimes C^{0} \stackrel{\sigma_{1}\left(D^{0}\right)}{\longrightarrow} T^{*} \otimes C^{1} \stackrel{\tau^{1}}{\rightarrow} C^{2}
$$

is exact. But (1.6.13) is the top row of the diagram used in defining $\rho^{1}: J_{1}\left(C^{1}\right) \rightarrow C^{2}$. We have therefore defined the operator $D^{1}: C^{1} \rightarrow C^{2}$.

It remains to show formal exactness. For this we consider the following diagram:

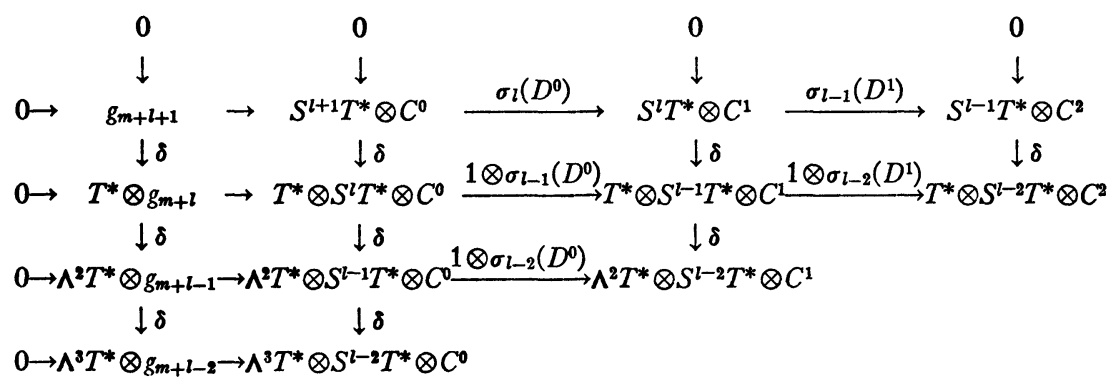

The columns are exact. For $l=2$ the second and third rows are exact by (1.6.12) and (1.6.13), and the first row is exact except, possibly, at $S^{2} T^{*} \otimes C^{1}$. We infer, by the usual diagram chase, that exactness of the first row at $S^{2} T^{*} \otimes C^{1}$ is equivalent to the exactness of the first column at $\Lambda^{2} T^{*} \otimes g_{m+1}$, and hence the first row is exact for $l=2$. Arguing by recursion, we conclude that, for $l \geqq 2$, the sequence

(1.6.14) $\quad 0 \rightarrow g_{m+l} \rightarrow S^{l} T^{*} \otimes C^{0} \rightarrow S^{l-1} T^{*} \otimes C^{1} \stackrel{\sigma_{l-2}\left(D^{1}\right)}{\longrightarrow} S^{l-2} T^{*} \otimes C^{2}$

is exact. 
Finally, consider the commutative diagram

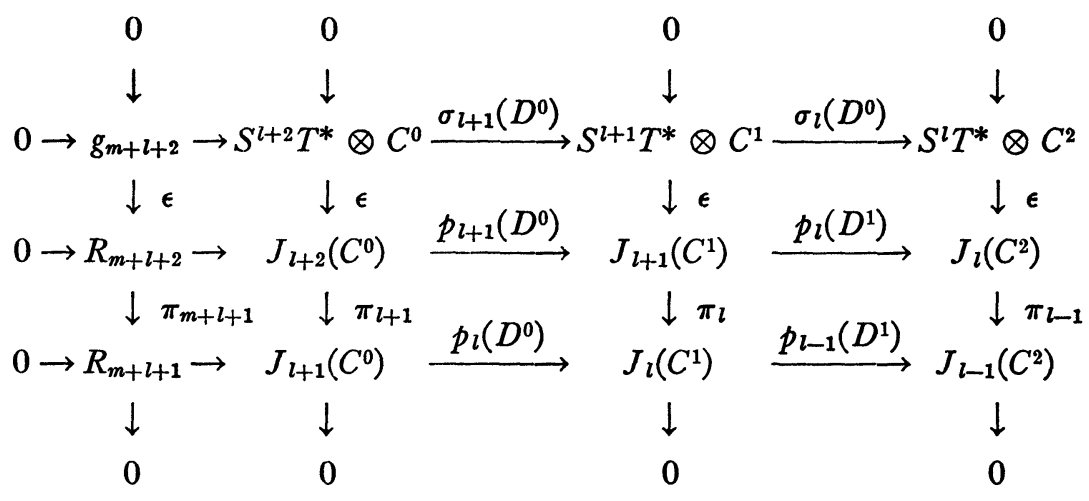

The columns are exact, and the first row is exact by (1.6.14). For $l=1$ the third row is exact by (1.6.11), and hence the second row is exact for $l=1$. Therefore, we establish inductively the exactness, for $l \geqq 2$, of the sequences

$$
0 \rightarrow R_{m+l} \rightarrow J_{l}\left(C^{0}\right) \stackrel{p_{l-1}\left(D^{0}\right)}{\longrightarrow} J_{l-1}\left(C^{1}\right) \stackrel{p_{l-2}\left(D^{1}\right)}{\longrightarrow} J_{l-2}\left(C^{2}\right) .
$$

Continuing in this fashion, we prove the existence of the complex (1.6.6) with properties (i), (ii) and (iii). Since each of the $C^{i}$ is isomorphic to a quotient $Q_{i-1}$, it is clear from condition (i) that the complex is unique up to isomorphisms.

The following proposition is easily verified:

Proposition 1.6.1. The cohomology of the second sequence (with $m \geqq \mu)$ is the same as the stable cohomology of the first sequence.

The relationship between the complex (1.5.2) of Theorem 1.5.7 and the first sequence is expressed by the diagram on the top of the following page. We infer, by means of the usual diagram chase, that the following proposition holds:

Proposition 1.6.2 (Quillen [19]). The cohomology of the complex (1.5.2) is isomorphic to the stable cohomology of the first sequence.

The exactness of the complex (1.5.2) at $G_{0}=F$ is equivalent to the local solvability of the inhomogeneous system of linear partial differential equations. In fact, given a local section $f$ of $G_{0}=F$ satisfying the "compatibility condition" $D_{1} f=0$, the exactness of (1.5.2) at $G_{0}=F$ implies the existence of a section $e$ of $E$, defined over some sufficiently small neighborhood, which satisfies $D e=D_{0} e=f$ where $D: E \rightarrow F$ is the 


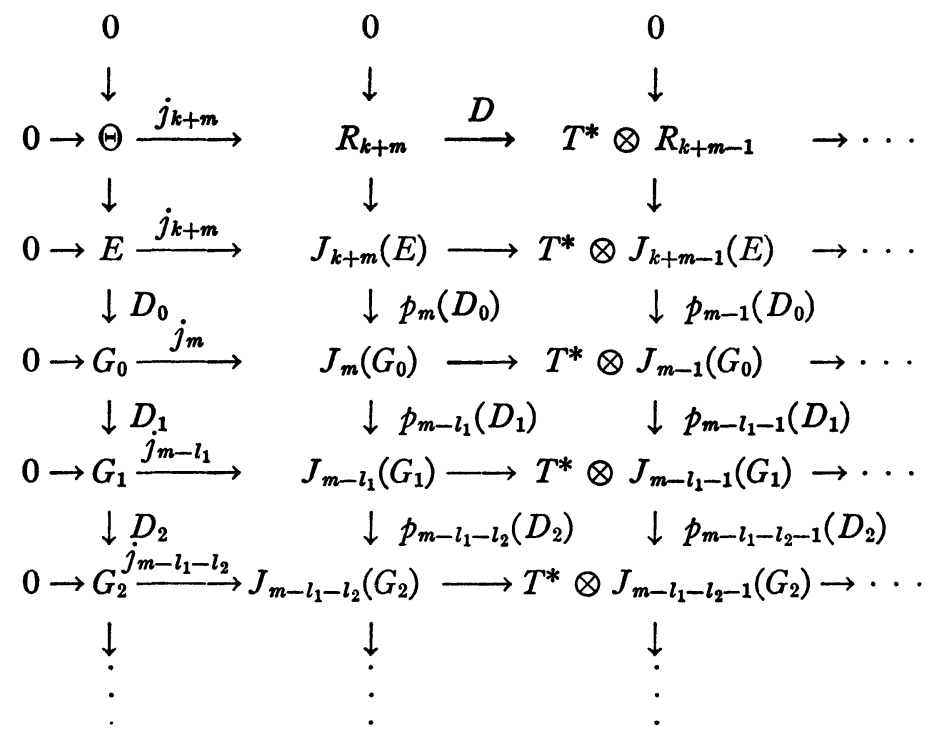

given differential operator from $E$ to $F$. From Propositions 1.6.1 and 1.6.2 we obtain:

TheOREM 1.6.2. Let $D: E \rightarrow F$ be a formally integrable differential operator. Then there exists a vector bundle $G_{1}$ and differential operator $D_{1}: F \rightarrow G_{1}$ such that the complex

$$
E \stackrel{D}{\rightarrow} F \stackrel{D_{1}}{\rightarrow} G_{1}
$$

is exact (local solvability of the inhomogeneous system) if and only if the second sequence attached to the given operator is exact at $C^{1}=C_{m}^{1}$ where $m \geqq \mu$ (the integer of Theorem 1.4.1).

It is well known that there exist systems of equations, i.e., differential operators $D: E \rightarrow F$, such that the sequence (1.6.15) is not exact in the $C^{\infty}$-sense, and these systems may even be determined. The first example of a determined system for which (1.6.15) (with $G_{1}=0$ and $D_{1}=0$ ) is not exact was given by $H$. Lewy [15], namely, the following inhomogeneous system of two equations in three independent variables $x_{1}, x_{2}, x_{3}$ and two unknown functions $f_{1}, f_{2}$ :

$$
\begin{aligned}
& \frac{\partial u_{1}}{\partial x_{1}}-\frac{\partial u_{2}}{\partial x_{2}}-2 x_{2} \frac{\partial u_{1}}{\partial x_{3}}-2 x_{1} \frac{\partial u_{2}}{\partial x_{3}}=f_{1}, \\
& \frac{\partial u_{1}}{\partial x_{1}}+\frac{\partial u_{2}}{\partial x_{2}}+2 x_{1} \frac{\partial u_{1}}{\partial x_{3}}-2 x_{2} \frac{\partial u_{2}}{\partial x_{3}}=f_{2} .
\end{aligned}
$$


Writing $u=u_{1}+i u_{2}, z=x_{1}+i x_{2}, f=f_{1}+i f_{2}$ and $\partial / \partial \bar{z}=\left(\partial / \partial x_{1}+i \partial / \partial x_{2}\right) / 2$, where $i=\sqrt{-1}$, we see that this system takes the simple form

$$
\partial u / \partial \bar{z}+i z\left(\partial u / \partial x_{3}\right)=\frac{1}{2} f .
$$

If we take $f_{2}=0$ and $f_{1}=f_{1}\left(x_{3}\right)$ to be a real-valued (differentiable) function of $x_{3}$, there is no local solution of (1.6.16) unless $f_{1}\left(x_{3}\right)$ is a real-analytic function of $x_{3}$, in which case a local analytic solution always exists.

For purposes of calculation it is sometimes convenient to have a more explicit representation of the second sequence. We suppose that $m \geqq \mu$ (integer of Theorem 1.4.1) and choose a splitting of the exact sequence

$$
0 \rightarrow \Lambda^{i} T^{*} \otimes g_{m} \rightarrow \Lambda^{i} T^{*} \otimes R_{m} \stackrel{\pi_{m-1}}{\rightarrow} \Lambda^{i} T^{*} \otimes R_{m-1} \rightarrow 0,
$$

namely a map

$$
P: \bigwedge^{i} T^{*} \otimes R_{m-1} \rightarrow \bigwedge^{i} T^{*} \otimes R_{m}
$$

satisfying $\pi_{m-1} \circ P=1$ (identity). Letting $Q=1-P \circ \pi_{m-1}$, we have the following commutative diagram:

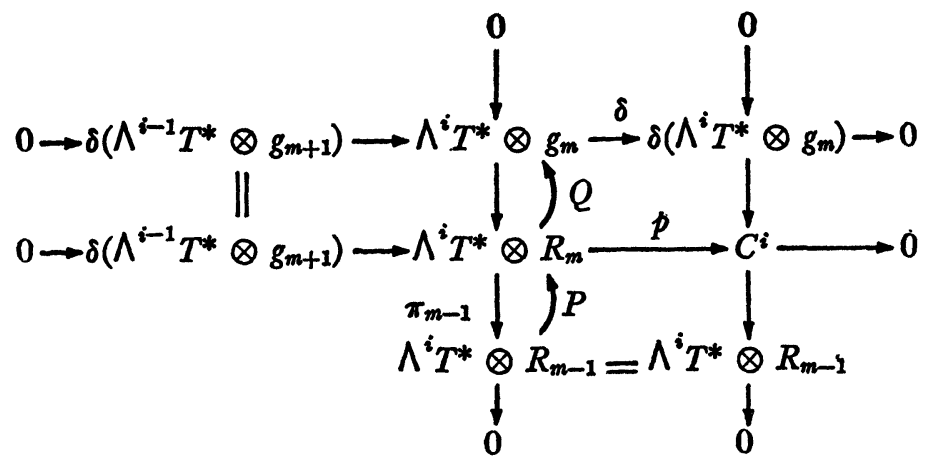

Here the last column is induced and is exact. We assert that the map

$$
\Lambda^{i} T^{*} \otimes R_{m} \rightarrow\left(\Lambda^{i} T^{*} \otimes R_{m-1}\right) \oplus \delta\left(\Lambda^{i} T^{*} \otimes g_{m}\right)
$$

sending $\sigma$ into $\left(\pi_{m-1} \sigma, \delta Q \sigma\right)$ induces, for each $i$, an isomorphism

$$
C^{i} \rightarrow\left(\Lambda^{i} T^{*} \otimes R_{m-1}\right) \oplus \delta\left(\Lambda^{i} T^{*} \otimes g_{m}\right) .
$$

If $\sigma \in \delta\left(\bigwedge^{i-1} T^{*} \otimes g_{m+1}\right)$, then $\pi_{m-1} \sigma=\delta Q \sigma=0$ and it follows that (1.6.18) is well defined. To prove injectivity, let $\sigma \in \Lambda^{i} T^{*} \otimes R_{m}$ satisfy $\pi_{m-1} \sigma=\delta Q \sigma=0$. Since $\sigma=P \pi_{m-1} \sigma+Q \sigma=Q \sigma$, we have $\pi_{m-1} \sigma=\delta \sigma=0$ and hence, by Theorem 1.4.1 (since $m \geqq \mu), \sigma \in \delta\left(\bigwedge^{i-1} T^{*} \otimes g_{m+1}\right)$ 
$=$ ker $p$. To prove surjectivity, let $\tau \in \Lambda^{i} T^{*} \otimes R_{m-1}, \zeta \in \delta\left(\Lambda^{i} T^{*} \otimes g_{m}\right)$, choose $\eta \in \Lambda^{i} T^{*} \otimes g_{m}$ such that $\zeta=\delta \eta$, and observe that (1.6.18) maps $p(P \tau+\eta)$ into $(\tau, \zeta)$.

Finally, we verify easily that, in terms of the isomorphism (1.6.18), the operator $D^{i}$ (see [21(b), §4]) is given by

$$
D^{i}(\sigma, \zeta)=(D P \sigma-\zeta, D P(D P \sigma-\zeta))
$$

where $D$ is the operator of the first sequence and where, as is easily seen, $(D P)^{2}=(D P) \circ(D P)$ is linear over functions. For simplicity, we set $D_{0}=D P$ and this formula becomes

$$
D^{i}(\sigma, \zeta)=\left(D_{0} \sigma-\zeta, D_{0}\left(D_{0} \sigma-\zeta\right)\right)
$$

where $D_{0}$ is a connection and $D_{0}^{2}$ its curvature (compare Bott [1], Cenkl [4]).

Henceforth, whenever no confusion will arise, we drop the superscripts on the operators of the second sequence and write it simply in the form

$$
0 \rightarrow \Theta \stackrel{j_{m}}{\rightarrow} C^{0} \stackrel{D}{\rightarrow} C^{1} \stackrel{D}{\rightarrow} C^{2} \stackrel{D}{\rightarrow} \ldots \stackrel{D}{\rightarrow} C^{n} \rightarrow 0
$$

where $C^{i}=C_{m}^{i}$ is defined by (1.6.3) and $m$ is a fixed integer, $m \geqq \mu$ (integer of Theorem 1.4.1).

1.7. Symbol sequences and characteristics. Let $T$ be the tangent bundle of $X, S^{l} T$ the $l$ th symmetric power of $T$, and $S=\oplus_{l_{\Sigma} 0} S^{l} T$ the symmetric algebra of $T$ which can be regarded as the ring of polynomial functions on $T^{*}$. We shall restrict our attention to the fibres of all vector bundles over a point of $X$ and, for simplicity, shall use the same notation for a vector bundle and its fibre over this point.

We observe first that we have a natural pairing

$$
S^{l} T \otimes S^{l} T^{*} \rightarrow R
$$

which is given by

$$
\left\langle t_{1} \cdots \cdot t_{l}, \xi^{l}\right\rangle=l ! \prod_{i=1}^{l}\left\langle t_{i}, \xi\right\rangle
$$

where $t_{i} \in T$ and $\xi \in T^{*}$ or, more generally, by

$$
\left\langle t_{1} \cdots t_{l}, \xi_{1} \cdots \xi_{l}\right\rangle=\sum_{\sigma} \prod_{i=1}^{l}\left\langle t_{i}, \xi_{\sigma(i)}\right\rangle
$$

where $\xi_{j} \in T^{*}$ and the summation in the right member of this equation is extended over all permutations of the integers $1,2, \cdots, l$. We 
identify $S^{l} T^{*}$ and the dual of $S^{l} T$ under this pairing. Then $\delta: S^{l} T^{*}$ $\rightarrow T^{*} \otimes S^{l-1} T^{*}$ has an adjoint map $\delta^{*}: T \otimes S^{l-1} T \rightarrow S^{l} T$ which is described as follows. Since $\delta \xi^{l}=l \xi \otimes \xi^{l-1} \in T^{*} \otimes S^{l-1} T^{*}$, the map $\delta^{*}$ satisfies

$$
\begin{aligned}
\left\langle\delta^{*}\left(t_{1} \otimes\left(t_{2} \cdots \cdot t_{l}\right)\right), \xi^{l}\right\rangle & =\left\langle t_{1} \otimes\left(t_{2} \cdots t_{l}\right), \delta \xi^{l}\right\rangle \\
& =\left\langle t_{1} \otimes\left(t_{2} \cdots t_{l}\right), l \xi \otimes \xi^{l-1}\right\rangle \\
& =l\left\langle t_{1}, \xi\right\rangle\left\langle t_{2} \cdots \cdot t_{l}, \xi^{l-1}\right\rangle \\
& =l ! \prod_{i=1}^{l}\left\langle t_{i}, \xi\right\rangle \\
& =\left\langle t_{1} \cdots \cdots t_{l}, \xi^{l}\right\rangle .
\end{aligned}
$$

Hence

$$
\delta^{*}\left(t_{1} \otimes\left(t_{2} \cdots \cdot t_{l}\right)\right)=t_{1} \cdot t_{2} \cdots \cdot t_{l},
$$

i.e., $\delta^{*}$ is the natural multiplication map.

We remark also that the value of $P \in S^{l} T$, regarded as a homogeneous polynomial of degree $l$, at a cotangent vector $\xi \in T^{*}$, is $P(\xi)$ $=(1 / l !)\left\langle P, \xi^{l}\right\rangle$. For $l \geqq 1$, let

$$
i_{l}(\xi): E \rightarrow S^{l} T^{*} \otimes E
$$

be the map sending the element $e$ of $E$ into $\left(\xi^{l} \otimes e\right) / l$ !. Then

$$
i_{l}(\xi): S^{l} T \otimes E \rightarrow E^{*}
$$

maps $P \otimes e^{*} \in S^{\imath} T \otimes E^{*}$ into $P(\xi) e^{*}$. In fact

$$
\begin{aligned}
\left\langle e, i_{l}(\xi) *\left(P \otimes e^{*}\right)\right\rangle & =\left\langle i_{l}(\xi) e, P \otimes e^{*}\right\rangle=\frac{1}{l !}\left\langle\xi^{l} \otimes e, P \otimes e^{*}\right\rangle \\
& =\frac{1}{l !}\left\langle P, \xi^{l}\right\rangle\left\langle e, e^{*}\right\rangle \\
& =P(\xi) \cdot\left\langle e, e^{*}\right\rangle .
\end{aligned}
$$

Now let $D: E \rightarrow F$ be a differential operator corresponding to the bundle morphism $\phi: J_{k}(E) \rightarrow F$ with $\operatorname{ker} \phi=R_{k}$. Consider, for $l \geqq 0$, the maps (induced by $\phi$ )

$$
\sigma_{l}(D)=\sigma_{l}(\phi): S^{k+l} T^{*} \otimes E \rightarrow S^{l} T^{*} \otimes F .
$$

If $\xi \in T^{*}$, we denote by

$$
\sigma_{\xi}(D)=\sigma_{\xi}(\phi): E \rightarrow F
$$

the composition $\sigma(D) \circ i_{k}(\xi)=\sigma_{0}(\phi) \circ i_{k}(\xi)$. The direct sum of the 
maps $\sigma_{l}(D)^{*}, l \geqq 0$, is a linear map

$$
\sigma^{*}(D): S \otimes F^{*} \rightarrow S \otimes E^{*}
$$

of graded vector spaces of degree $k$. From the commutativity of diagram (1.4.3), we infer that $\sigma^{*}(D)$ commutes with multiplication by $t \in T$ and hence that $\sigma^{*}(D)$ is an $S$-module morphism. Let $M$ be the cokernel of $\sigma^{*}(D)$, i.e.,

$$
S \otimes F^{*} \stackrel{\sigma^{*}(D)}{\longrightarrow} S \otimes E^{*} \rightarrow M \rightarrow 0 .
$$

We have the isomorphism

$$
M \cong \underset{l \geq-k}{\otimes} g_{k+l}^{*}
$$

and we call $M$ the symbol module of the differential operator.

Definition 1.7.1. We say that a cotangent vector $\xi$ is characterstic (for $D$ ) if $\sigma_{\xi}(D)$ fails to be injective. The operator $D$ is elliptic if it has no nonzero real characteristic cotangent vectors.

In order for $\xi$ to be characteristic, a finite number of algebraic equations involving $\xi$ must hold. The set of all characteristic covectors at the point $x$ of $X$ forms an algebraic variety called the characteristic variety at $x$ and we shall denote it by $V_{x}$. For simplicity, we shall call the set of all characteristic vectors, at all points of $X$, the characteristic variety of $D$ and shall denote it by $V$. Thus $V$ is a subset of $T^{*}$ and, for each point $x$ of $X, V \cap T_{x}^{*}=V_{x}$. Therefore, by abuse of notation, we are calling a "family of characteristic varieties" simply a "characteristic variety." However, we continue to focus our attention on the fibres of vector bundles over an arbitrary, but fixed, point of $X$.

We shall now bring together various equivalent criteria for a covector $\xi$ to be characteristic.

Proposition 1.7.1. A covector $\xi \in T^{*}$ is characteristic if and only if there is a nonzero $e \in E$ such that $\xi^{k} \otimes e \in g_{k}$, where $k$ is the order of the operator.

This proposition is a consequence of the exactness of the sequence

$$
0 \rightarrow g_{k} \rightarrow S^{k} T^{*} \otimes E \stackrel{\sigma(D)}{\longrightarrow} F .
$$

Suppose that $D$ is a first-order differential operator, i.e., $k=1$. Then we can identify $T^{*} \otimes E$ with $\operatorname{Hom}(T, E)$ and, if $\xi \neq 0$, we can regard $\xi \otimes e$ as the linear transformation of rank 1 which sends $t \in T$ into $\langle t, \xi\rangle e$. 
Proposition 1.7.2. A covector $\xi \in T^{*}$ is characteristic if and only if there is an $e \in E, e \neq 0$, such that $\xi^{k+l} \otimes e \in g_{k+l}$ for some or all $l \geqq 0$.

Proof. By the commutativity of diagram (1.4.3) we have, for $\xi \in T^{*}, e \in E$,

$$
\begin{aligned}
\delta \sigma_{l+1}(D)\left(\xi^{k+l+1} \otimes e\right) & =\sigma_{l}(D) \delta\left(\xi^{k+l+1} \otimes e\right) \\
& =(k+l+1) \sigma_{l}(D)\left(\xi \otimes \xi^{k+l} \otimes e\right) \\
& =(k+l+1) \xi \otimes \sigma_{l}(D)\left(\xi^{k+l} \otimes e\right) .
\end{aligned}
$$

Since $\delta: S^{m} T^{*} \rightarrow T^{*} \otimes S^{m-1} T^{*}$ is injective for $m \geqq 1$, we see that $\sigma_{l+1}(D)\left(\xi^{k+l+1} \otimes e\right)$ vanishes if and only if $\sigma_{l}(D)\left(\xi^{k+l} \otimes e\right)$ vanishes.

Following Guillemin, Quillen and Sternberg (see [10], [22]), we introduce the characteristic ideal.

DEFinition 1.7.2. The characteristic ideal $J \subset S$ of $V$ (characteristic variety) is the annihilator of $M$.

Thus a homogeneous section $P$ of $S$ takes values in $J$ if, for each $x \in X, P_{x} M_{x}=0$.

Definition 1.7.3. We say that a differential operator $D: E \rightarrow F$ is of finite type if $g_{m}=0$ for all sufficiently large $m$; otherwise we say that it is of infinite type.

We shall now give a criterion, due to Guillemin, Quillen and Sternberg (loc. cit.), in order that a differential operator be of finite type.

If $\xi$ is a cotangent vector, we denote by $\operatorname{Tr}(\xi)$ the set of polynomials $P$ of $S$ which vanish at $\xi$. Then $\operatorname{m(}(\xi)$ is a maximal ideal of $S$, and it is prime since $S / \mathscr{T}(\xi)$ is isomorphic to $R$ (real numbers). We recall that the localization of $S$ at the maximal ideal $\mathfrak{T}(\xi)$ is

$$
S_{\xi}=\left\{\frac{P}{Q} \mid P, Q \in S, Q \notin \mathfrak{N}(\xi)\right\},
$$

where the fractions $P / Q, P^{\prime} / Q^{\prime}$ are identified if there exists $Q^{\prime \prime} \in S$, $Q^{\prime \prime} \notin \operatorname{Tr}(\xi)$, such that $Q^{\prime \prime}\left(Q^{\prime} P-Q P^{\prime}\right)=0$.

Proposition 1.7.3. Let $\xi \in T^{*}$ be a nonzero covector. Then $\xi$ is noncharacteristic if and only if $M_{\xi}=M \otimes_{S} S_{\xi}=0$, where $S_{\xi}$ is the localization of $S$ at the maximal ideal $\mathfrak{M ( \xi )}$.

For a proof see Goldschmidt [6(a)], Proposition 6.3.

It follows immediately from the definitions that Proposition 1.7.3 is equivalent to the following:

Proposition 1.7.4. A covector $\xi$ is characteristic if and only if $P(\xi)=0$ for all $P \in J$. 
In fact, a nonzero covector $\xi$ is noncharacteristic if and only if there exists $Q \in J$ satisfying $Q(\xi) \neq 0$.

Definition 1.7.4. An element of $C \otimes T^{*}$ is called a complex characeristic if it is a zero of the ideal $J$.

Proposition 1.7.5. A differential operator is of infinite type if and only if it has nonzero complex characteristics.

Proposition 1.7.5 is an immediate consequence of Proposition 1.7.3 and a theorem of J.-P. Serre [20] which asserts that, if $M_{\xi}=0$ for all $\xi$, then $M_{m}=g_{m}^{*}=0$ for all sufficiently large $m$.

A simple example of an operator of finite type is provided by the following equations

$$
\partial e_{j} / \partial x^{k}+\partial e_{k} / \partial x^{j}=0, \quad j, k=1,2, \cdots, n,
$$

where $e=\left(e_{1}, \cdots, e_{n}\right)$ is a local section of $E=R^{n} \times R^{n}$ depending differentiably on the coordinate $x=\left(x_{1}, \cdots, x_{n}\right)$ of $R^{n}$. The complex covector $\xi=\left(\xi_{1}, \cdots, \xi_{n}\right)$ is characteristic if

$$
\xi_{k} e_{j}+\xi_{j} e_{k}=0, \quad j, k=1,2, \cdots, n,
$$

where $e=\left(e_{1}, \cdots, e_{n}\right) \neq 0$, and we infer immediately that $\xi=0$. A trivial calculation shows that $g_{m}=0$ for $m \geqq 2$.

The following theorems are due to Quillen [19].

THEOREM 1.7.1. Let

$$
E_{0} \stackrel{D_{0}}{\longrightarrow} E_{1} \stackrel{D_{1}}{\longrightarrow} E_{2} \stackrel{D_{2}}{\longrightarrow} \cdots \rightarrow E_{m} \rightarrow 0
$$

be a complex of first-order differential operators where the $E_{i}$ are vector bundles. Suppose that this complex is formally exact (in the sense of (iii), Theorem 1.6.1) and that the symbol maps

$$
\sigma\left(D_{i}\right): T^{*} \otimes E_{i} \rightarrow E_{i+1}
$$

are surjective. Then, if $\xi \in T^{*}$ is noncharacteristic for the operator $D_{0}$, the sequence

$$
0 \rightarrow E_{0} \stackrel{\sigma_{\xi}\left(D_{0}\right)}{\longrightarrow} E_{1} \stackrel{\sigma_{\xi}\left(D_{1}\right)}{\longrightarrow} E_{2} \stackrel{\sigma_{\xi}\left(D_{2}\right)}{\longrightarrow} \cdots \rightarrow E_{m} \rightarrow 0
$$

is exact.

ThEOREM 1.7.2. Let $D: E \rightarrow F$ be a formally integrable differential operator of order $k$, and let $C^{i}=C_{m}^{i}$ be defined by (1.6.3) (second complex attached to a differential operator) where $m \geqq \mu$ (integer of Theorem 1.4.1). If $\xi \in T^{*}$ is a nonzero cotangent vector, the following assertions 
are equivalent:

(i) $\xi$ is noncharacteristic;

(ii) the sequence

$$
0 \rightarrow C^{0} \stackrel{\sigma_{\xi}\left(D^{0}\right)}{\longrightarrow} C^{1}
$$

is exact;

(iii) the sequence

$$
0 \rightarrow C^{0} \stackrel{\sigma_{\xi}\left(D^{0}\right)}{\longrightarrow} C^{1} \stackrel{\sigma_{\xi}\left(D^{1}\right)}{\longrightarrow} C^{2} \stackrel{\sigma_{\xi}\left(D^{2}\right)}{\longrightarrow} \cdots \rightarrow C^{n} \rightarrow 0
$$

is exact.

The hypotheses in Theorem 1.7.2 can be weakened. It is sufficient to assume (see Goldschmidt [6(a) ]) that the $C^{i}=C_{m}^{i}$ are defined by (1.6.3) where $m \geqq \mu$ and that the symbol maps are exterior multiplication by $\xi$ followed by passage to the quotient.

Since the second sequence attached to $D$ is a complex which, by Theorem 1.6.1, satisfies the hypotheses of Theorem 1.7.1, the equivalence of (ii) and (iii) in Theorem 1.7.2 follows from Theorem 1.7.1. On the other hand, the equivalence of (ii) and (iii) is almost immediate. Theorems 1.7.1 and 1.7.2 can be proved by standard arguments from the theory of commutative algebra (see Quillen [19], Goldschmidt [6(a)] and Sternberg [22]). We shall omit a proof of Theorem 1.7.1 and shall prove instead Theorem 1.7.2, which is essentially equivalent to the following theorem.

Let $\xi \in T^{*}$ be a nonzero covector, denote by $H$ the subspace of $T$ which is annihilated by $\xi$, and denote by $H^{*}$ the restriction of $T^{*}$ to $H$. Now $g_{l}$ is a subspace of Hom $\left(S^{\imath} T, E\right)$ and we denote by $h_{l}$ the restriction of $g_{l}$ to $S^{l} H$.

THEOREM 1.7.3. Let $\xi$ be a noncharacteristic covector. Then the restriction maps $g_{l} \rightarrow h_{l}$ are bijective for $l \geqq k$, and the sequence

$$
\Lambda^{i-2} H^{*} \otimes h_{m+1} \stackrel{\delta}{\rightarrow} \Lambda^{-1} H^{*} \otimes h_{m} \rightarrow \Lambda^{i} H^{*} \otimes h_{m-1}
$$

is exact for $m \geqq \mu$ and $i \geqq 1$.

Suppose that the hypotheses of Theorem 1.7.2 are satisfied, and consider the second sequence of Theorem 1.6.1. For simplicity we write

$$
R_{m}^{i}=\Lambda^{i} T^{*} \otimes R_{m}, \quad \alpha g_{m}^{i+1}=\delta\left(\Lambda^{i} T^{*} \otimes g_{m+1}\right) .
$$

We choose a splitting (1.6.7) (with $m$ replaced by $m+1$, for conve- 
nience) and then, by the isomorphism (1.6.18), we can make the identification

$$
C^{i}=C_{m+1}^{i}=R_{m}^{i} \oplus \alpha g_{m}^{i+1} .
$$

Let $x_{0}$ be an arbitrary point of $X$, and let $\xi$ be a nonzero cotangent vector at $x_{0}$. We choose a coordinate $x=\left(x^{1}, \cdots, x^{n}\right)$ on a neighborhood $U$ of $x_{0}$ such that the restrictions $R_{m}^{i} \mid U$ and $\alpha g_{m}^{i+1} \mid U$ are trivial bundles; then $C^{i} \mid U$ is also trivial. By (1.6.19), the principal part of the operator $D^{i}: C^{i} \rightarrow C^{i+1}$ is given by

$$
(\sigma, \zeta) \rightarrow\left(\sum_{\nu=1}^{n} d x^{\nu} \wedge \partial_{\nu} \sigma,-\sum_{\nu=1}^{n} d x^{\nu} \wedge \partial_{\nu} \zeta\right),
$$

where $\partial_{\nu}=\partial / \partial x^{\nu}$. Thus the symbol sequence (1.7.4) at $\xi$ is the direct sum of the sequences

$$
\begin{gathered}
0 \rightarrow R_{m} \stackrel{\xi}{\rightarrow} R_{m}^{1} \stackrel{\xi}{\rightarrow} R_{m}^{2} \stackrel{\xi}{\rightarrow} \ldots \stackrel{\xi}{\rightarrow} R_{m}^{n} \rightarrow 0, \\
0 \rightarrow \alpha g_{m}^{1} \stackrel{-\xi}{\rightarrow} \alpha g_{m}^{2} \stackrel{-\xi}{\rightarrow} \alpha g_{m}^{3} \stackrel{-\xi}{\rightarrow} \ldots \stackrel{-\xi}{\rightarrow} \alpha g_{m}^{n} \rightarrow 0,
\end{gathered}
$$

where the maps labelled $\xi$ and $-\xi$ denote left exterior multiplication by the covectors $\xi,-\xi$. The sequence (1.7.6) is always exact (for any nonzero covector $\xi$ ), so Theorem 1.7.2 is equivalent to the following theorem:

THEOREM 1.7.4. The sequence (1.7.7) is exact for $m \geqq \mu$ (the integer of Theorem 1.4.1) if and only if $\xi$ is noncharacteristic for $D$.

It is therefore sufficient to exhibit the relationship between Theorems 1.7.3 and 1.7.4. Let $\alpha h_{m}^{l}$ denote the restriction of $\alpha g_{m}^{l}$ to $\Lambda^{l} H \otimes S^{l} H$ or, equivalently, the kernel of the map

$$
\delta: \Lambda^{l} H^{*} \otimes h_{m} \rightarrow \Lambda^{l+1} H^{*} \otimes h_{m-1} .
$$

Consider diagram $(1.78)_{l}$ (where $m \geqq \mu$ ) on the top of the following page. This diagram is commutative, and it is exact except for the last row and the first and last columns.

Suppose that the restriction maps

$$
g_{m+l} \rightarrow h_{m+l}
$$

are isomorphisms for $l \geqq 1$. Then the first column of diagram (1.7.8) is exact, and a diagram chase shows that the last row is exact if and only if the last column is. The surjectivity of the map

$$
\alpha g_{m}^{l} \rightarrow \alpha h_{m}^{l}
$$


$(1.7 .8)_{\imath}$
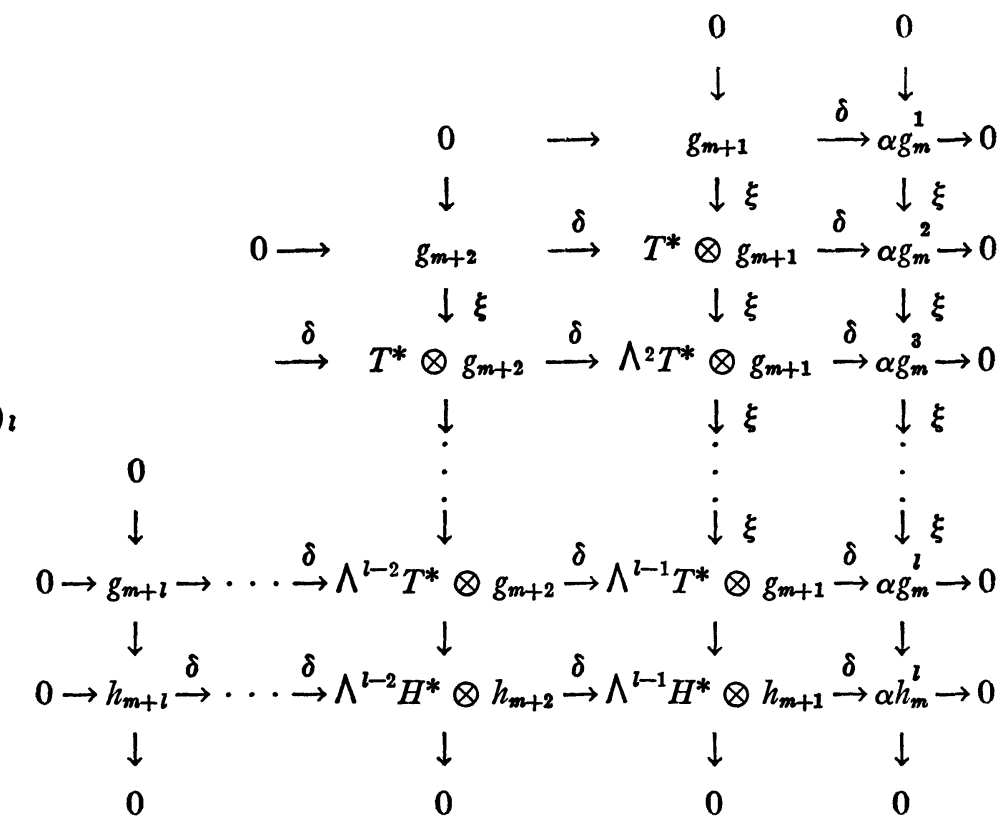

is equivalent to the surjectivity of the map

$$
\delta: \wedge^{l-1} H^{*} \otimes h_{m+1} \rightarrow \alpha h_{m}^{l}
$$

and the surjectivity of (1.7.11) is equivalent to the exactness of the sequence (1.7.5). On the other hand, it can be seen by recursion that the exactness of (1.7.7) implies the surjectivity of (1.7.10).

We now prove Theorem 1.7.3, and we follow Sweeney [23 (b)].

We show first that the maps (1.7.9) are isomorphisms and we may suppose, without loss of generality, that the order $k$ of the differential operator is 1 . We may also assume, by suitable choice of a local coordinate $x=\left(x^{1}, \cdots, x^{n}\right)$ on $X$, that $\xi=d x^{n}$. An element $\sigma$ of $g_{l}$, expressed in terms of the local coordinate, can be written in the form

$$
\sigma=\sum_{|\alpha|=l} \sigma_{\alpha} d x^{\alpha} / \alpha !
$$

where. if $\alpha=\left(\alpha_{1}, \cdots, \alpha_{n}\right), d x^{\alpha}=\left(d x^{1}\right)^{\alpha_{1}}\left(d x^{2}\right)^{\alpha_{2}} \cdots\left(d x^{n}\right)^{\alpha_{n}}$, and $\sigma_{\alpha} \in E$. Then (compare $\$ 1.3$ )

$$
\delta=\sum_{i=1}^{n} d x^{i} \wedge \delta_{i}
$$


where

$$
\delta_{i} \sigma=\sum_{|\alpha|=l-1} \sigma_{\alpha+1_{i}} d x^{\alpha} / \alpha !
$$

Let $\phi: g_{l} \rightarrow h_{l}$ be the restriction map; then

$$
\phi(\sigma)=\sum_{\substack{|\alpha|=l \\ \alpha_{n}=0}} \sigma_{\alpha} d x^{\alpha} / \alpha ! .
$$

LEMMA 1.7.1. The map $\phi: g_{l} \rightarrow h_{l}$ is an isomorphism for each $l \geqq 1$.

Proof. Let $\sigma \in g_{l}, \sigma \neq 0$, and suppose that $\phi(\sigma)=0$. Among all multi-indices $\alpha$ with $|\alpha|=l$ and $\sigma_{\alpha} \neq 0$, choose a multi-index $\beta$ for which $\beta_{n}$ is minimal. Since $\phi(\sigma)=0$, we must have $\beta_{n} \geqq 1$ and, from the minimality, we infer that $\delta_{i} \delta^{\beta-1_{n}} \sigma=0$ for $i=1,2, \cdots, n-1$, where, if $\gamma=\left(\gamma_{1}, \cdots, \gamma_{n}\right), \delta^{\gamma}=\delta_{1} \gamma_{1} \cdots \delta_{n} \gamma_{n}$. Thus $\delta^{\beta-1_{n} \sigma}$ is a nonzero element of $g_{1}$ depending only on $d x^{n}$ and hence of the form $d x^{n} \otimes e$, $e \neq 0$, in contradiction of the assumption that $\xi=d x^{n}$ is noncharacteristic.

Finally we show that (1.7.5) is exact, and we reduce the exactness to Theorem 1.4.1 ( $\delta$-Poincaré lemma for the $g_{l}$ ) by proving that, for all sufficiently large $l, h_{l+1}$ is the prolongation of $h_{l}$.

Introduce on $S^{l} H^{*} \otimes E$ the scalar product

$$
\left\langle\sum \sigma_{\alpha} d x^{\alpha} / \alpha !, \quad \sum \tau_{\alpha} d x^{\alpha} / \alpha !\right\rangle=\sum\left\langle\sigma_{\alpha}, \tau_{\alpha}\right\rangle / \alpha !
$$

where $\left\langle\sigma_{\alpha}, \tau_{\alpha}\right\rangle$ is the scalar product of a metric along the fibres of the vector bundle $E$. Then the adjoint of $\delta_{i}$ is multiplication by $d x^{i}$. Since $\delta_{i}\left(h_{l+1}\right) \subset h_{l}$ for $i=1,2, \cdots, n-1$, the orthogonal complement $h^{\prime}=\oplus h_{l}^{\prime}$ of $h$ is a submodule of the module of all $E$-valued polynomials in $d x^{1}, \cdots, d x^{n-1}$. Therefore, by the Hilbert basis theorem, there exists an integer $\mu^{\prime}$ such that, for $l \geqq \mu^{\prime}$, we have $\sigma \in h_{l+1}^{\prime}$ if and only if $\sigma=\sigma_{1} d x^{1}+\cdots+\sigma_{n} d x^{n}$ for some $\sigma_{1}, \cdots, \sigma_{n} \in h_{l}^{\prime}$. Thus, if a homogeneous polynomial satisfies $\delta_{i} \sigma \in h_{l}$ for $i=1,2, \cdots, n-1$, we have

$$
\sigma=\sum_{i}\left\langle\delta_{i} \sigma, h_{l}^{\prime}\right\rangle=\sum_{i}\left\langle\sigma, d x^{i} \cdot h_{l}^{\prime}\right\rangle=\left\langle\sigma, h_{l+1}^{\prime}\right\rangle,
$$

and we infer that $\sigma \in h_{l}$ if and only if $\delta \gamma \sigma \in h_{\mu^{\prime}}$ for each $\gamma,|\gamma|=l-\mu^{\prime}$. It follows that the spaces $h_{l}, l \geqq \mu^{\prime}$, are obtained from $h_{\mu^{\prime}}$ by prolongation. By Theorem 1.4.1 the sequence (1.7.5) is exact for $m \geqq \mu+\mu^{\prime}$, where $\mu$ is the integer of that theorem. Consider, finally, the diagram on the top of the following page. The middle row is clearly exact, and we conclude by a downward induction that (1.7.5) is exact for $m \geqq \mu$. This completes the proof of Theorem 1.7.3 and hence of Theorem 1.7.2. 


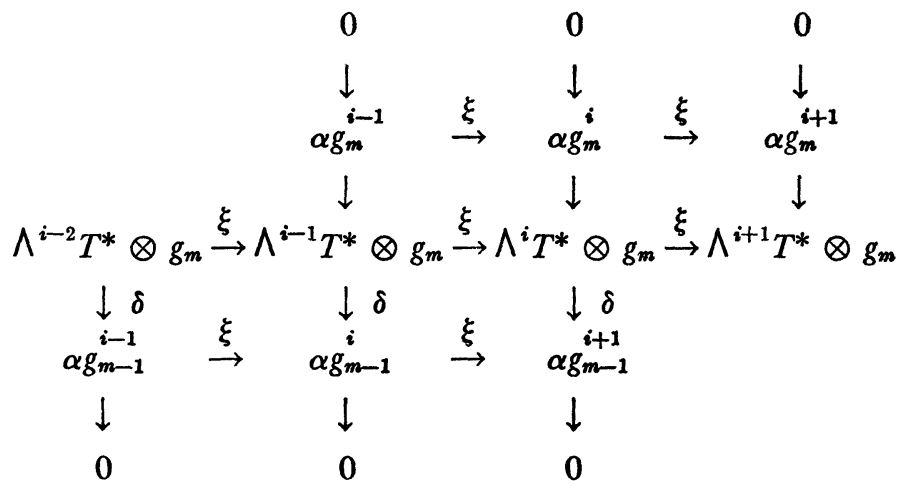

1.8. Guillemin's local decomposition and Cauchy problem. The results of this section are due to V. W. Guillemin [8] (see also [22], [16]).

We continue for the moment to restrict our attention to the fibres of all vector bundles over an arbitrary, but fixed, point of $X$ and to use the same notation for a vector bundle and its fibre over this point.

Let $D^{i}: C^{i} \rightarrow C^{i+1}$ be the $i$ th operator of the second sequence attached to a formally integrable differential operator of order $k$, where $C^{i}=C_{m}^{t}$ and $m \geqq \mu$ (integer of Theorem 1.4.1). We shall sometimes find it convenient to drop the superscript on $D^{i}$ and write simply $D$ for $D^{i}$. Now let $U$ be a subspace of $T^{*}$. Then $\delta: S^{l} U \rightarrow S^{l-1} U \otimes U$ can be regarded as a map $S^{l} U \rightarrow S^{l-1} U \otimes T^{*}$ followed by the restriction $U \rightarrow T^{*}$. We can consider the restriction of $\sigma_{l}(D)=\sigma_{l}\left(D^{i}\right)$ to $S^{l+1} U \otimes C^{i}$ and we obtain the map

$$
\sigma(D)=\sigma_{l}(D): S^{l+1} U \otimes C^{i} \rightarrow S^{l} U \otimes C^{i+1} .
$$

Definition 1.8.1. A subspace $U \subset T^{*}$ is called noncharacteristic if the map

$$
\sigma(D): U \otimes C^{0} \rightarrow C^{1}
$$

is injective, i.e., if

$$
\left(U \otimes C^{0}\right) \cap g_{m+1}=0
$$

where $g_{m+1}$ is the kernel of $\sigma(D): T^{*} \otimes C^{0} \rightarrow C^{1}$ (compare $\$ 1.6$ ).

Let $H=H_{U}$ be the subspace of $T$ which is annihilated by $U$, and denote by $H^{*}=H_{U}^{*}$ the restriction of $T^{*}$ to $H$. We denote by $h_{l}$ the restriction of $g_{l}\left(g_{l} \subset \operatorname{Hom}\left(S^{l} T, E\right)\right)$ to $S^{l} H$.

Guillemin (loc. cit.) has proved the following generalization of Theorems 1.7.2 and 1.7.3: 
THEOREM 1.8.1. Let $U \subset T^{*}$ be a noncharacteristic subspace. Then

(i) the restriction $m a p g_{l} \rightarrow h_{l}$ is bijective for $l \geqq k$;

(ii) the sequence

$$
\Lambda^{-2} H^{*} \otimes h_{m+1} \stackrel{\delta}{\rightarrow} \bigwedge^{i-1} H^{*} \otimes h_{m} \stackrel{\delta}{\rightarrow} \Lambda^{i} H^{*} \otimes h_{m-1}
$$

is exact for $m \geqq \mu$ (integer of Theorem 1.4.1) and $i \geqq 1$;

(iii) the sequence

$$
\begin{gathered}
0 \rightarrow S^{l+1} U \otimes C^{0} \stackrel{\sigma_{l}(D)}{\longrightarrow} S^{l} U \otimes C^{1} \\
\stackrel{\sigma_{l-1}(D)}{\longrightarrow} S^{l-1} U \otimes C^{2} \stackrel{\sigma_{l-2}(D)}{\longrightarrow} \cdots \stackrel{\sigma(D)}{\longrightarrow} C^{l+1} \rightarrow 0
\end{gathered}
$$

is exact.

The proof of (i) and (ii) is by induction on the dimension of $U$, and the case where $\operatorname{dim} U=1$ is Theorem 1.7.3. The assertion (iii) then follows from a diagram analogous to $(1.7 .8)_{l}$. We shall omit the details of the proof, and shall turn to an interesting application of Theorem 1.8.1 which has been made by Guillemin (see also Sternberg [22]).

Given a point $x_{0}$ of $X$, let $U_{x_{0}}$ be a maximal noncharacteristic subspace of $T_{x_{0}}^{*}$. We can choose a local coordinate $\left(x^{1}, x^{2}, \cdots, x^{n}\right)$ on a neighborhood of $x_{0}$ such that $d x^{1}, d x^{2}, \cdots, d x^{k}$ form a basis for $U_{x_{0}}$ at the point $x_{0}$. Then $d x^{1}, d x^{2}, \cdots, d x^{k}$ span a maximal noncharacteristic subspace $U_{x}$ of $T_{x}^{*}$, and $d x^{k+1}, d x^{k+2}, \cdots, d x^{n}$ span a complementary subspace $W_{x}$ of $T_{x}^{*}$, at each point $x$ of some neighborhood of $x_{0}$. Let $U$ denote the sub-bundle of $T^{*}$ (restricted to the neighborhood) which is spanned by $d x^{1}, d x^{2}, \cdots, d x^{k}$, and let $W$ be the sub-bundle spanned by the remaining differentials $d x^{k+1}, d x^{k+2}, \ldots, d x^{n}$. We can then regard (1.8.4) as a sequence of bundle maps.

Since $d x^{i}$ is noncharacteristic for $1 \leqq i \leqq k$, the map

$$
\sigma_{d x i}(D): C^{0} \rightarrow C^{1}
$$

is injective and we write

$$
E_{i}=\sigma_{d x^{i}}(D)\left(C^{0}\right), \quad i=1,2, \cdots, k .
$$

It is easily seen that we have the direct-sum decomposition

$$
C^{1}=E_{0} \oplus E_{1} \oplus \cdots \oplus E_{k}
$$

where $E_{0}$ is a complement of $E_{1} \oplus \cdots \oplus E_{k}$. We let

$$
\pi_{i}: C^{1} \rightarrow E_{i}, \quad i=0,1,2, \cdots, k
$$

be the projections, and we define 


$$
D_{0}=\pi_{0} D, \quad D_{i}=\sigma_{d x i}(D)^{-1} \circ \pi_{i} \circ D \quad(1 \leqq i \leqq k)
$$

where

$$
D_{0}: C^{0} \rightarrow E_{0}, \quad D_{i}: C^{0} \rightarrow C^{0} \quad(1 \leqq i \leqq k)
$$

are differential operators. Then

$$
D=D_{0}+\sum_{i=1}^{k} \sigma_{d x i}(D) D_{i}
$$

Moreover

$$
\begin{aligned}
& \sigma_{d x i}\left(D_{j}\right)=\delta_{i j} \cdot I, \\
& \sigma_{d x i}\left(D_{0}\right)=0,
\end{aligned} \quad i=1,2, \cdots, k,
$$

where $I$ is the identity map of $C^{0}$. In fact, from the definitions we have

$$
D_{i}=\partial / \partial x^{i}+L_{i}, \quad i=1,2, \cdots, k,
$$

where $L_{i}=L_{i}\left(\partial / \partial x^{k+1}, \cdots, \partial / \partial x^{n}\right)$ is an operator involving differentiations only along the sheets of the local foliation $x^{1}=$ constant, $x^{2}=$ constant, $\cdots, x^{k}=$ constant, and

$$
D_{0}=D_{0}\left(\partial / \partial x^{k+1}, \cdots, \partial / \partial x^{n}\right)
$$

is also an operator differentiating only along the sheets.

We now show that the operators (1.8.5) satisfy certain commutation relations. In the sequel we shall write $D$ for the operator $D^{0}: C^{0}$ $\rightarrow C^{1}$ and $D^{\prime}$ for $D^{1}: C^{1} \rightarrow C^{2}$. Then $D^{\prime} D=0$ and we shall show first that

$$
\sigma_{d x i}\left(D^{\prime}\right) D=-D^{\prime} \sigma_{d x i}(D), \quad i=1,2, \cdots, k .
$$

If $f$ is any function and $u$ any section of $C^{1}$, we have

$$
\sigma_{d f}\left(D^{\prime}\right) u=D^{\prime}(f u)-f D^{\prime} u .
$$

Hence, in particular,

$$
\sigma_{d x i}\left(D^{\prime}\right) u=D^{\prime}\left(x^{i} u\right)-x^{i} D^{\prime} u .
$$

Taking $u=D v$, where $v$ is a section of $C^{0}$, we obtain

$$
\sigma_{d x i}\left(D^{\prime}\right) D v=D^{\prime}\left(x^{i} D v\right)-x^{i} D^{\prime} D v=D^{\prime}\left(x^{i} D v\right)
$$

since $D^{\prime} D=0$. Since (1.8.12) also holds for $D$, we have

$$
D^{\prime}\left(x^{i} D v\right)=D^{\prime}\left(D\left(x^{i} v\right)-\sigma_{d x i}(D) v\right)=-D^{\prime}\left(\sigma_{d x i}(D) v\right)
$$

and it follows that $\sigma_{d x^{i}}\left(D^{\prime}\right) D v=-D^{\prime} \sigma_{d x^{i}}(D) v$. Since $v$ is arbitrary, 
formula (1.8.11) follows. Applying $\sigma_{d x^{i}}$ to (1.8.11), we obtain

$$
\sigma_{d x i}\left(D^{\prime}\right) \sigma_{d x j}(D)=-\sigma_{d x j}\left(D^{\prime}\right) \sigma_{d x i}(D) .
$$

Next, substituting from (1.8.7) into the left member of (1.8.11), we have

$$
\sigma_{d x i}\left(D^{\prime}\right)\left(\pi_{0} D+\sum_{j=1}^{k} \sigma_{d x^{j}}(D) D_{i}\right)=-D^{\prime} \sigma_{d x i}(D) .
$$

Multiplying this identity on the right by $D_{i}$ and summing on $i$, we obtain

$$
\begin{aligned}
\sum_{i=1}^{k} \sigma_{d x i}\left(D^{\prime}\right)\left(\pi_{0} D D_{i}+\sum_{j=1}^{k} \sigma_{d x^{j}}(D) D_{j} D_{i}\right) & =-D^{\prime} \sum_{i=1}^{k} \sigma_{d x i}(D) D_{i} \\
=-D^{\prime} \sum_{i=1}^{k} \pi_{i} D & =D^{\prime}\left(D_{0}-D\right)=D^{\prime} D_{0}
\end{aligned}
$$

Therefore, by (1.8.13),

$$
\begin{aligned}
\sum_{1 \leq i<j \leq k} \sigma_{d x i}\left(D^{\prime}\right) \sigma_{d x^{j}}(D)\left(D_{j} D_{i}-D_{i} D_{j}\right)+ & \sum_{i=1}^{k} \sigma_{d x i}\left(D^{\prime}\right) \pi_{0} D D_{i} \\
& =D^{\prime} D_{0} .
\end{aligned}
$$

For simplicity we write

$$
\sigma_{i}=\sigma_{d x i}(D), \quad \sigma_{i}^{\prime}=\sigma_{d x i}\left(D^{\prime}\right), \quad i=1,2, \cdots, k .
$$

Then (1.8.13) becomes

$$
\sigma_{i}^{\prime} \sigma_{j}=-\sigma_{j}^{\prime} \sigma_{i}, \quad 1 \leqq i, j \leqq k .
$$

We denote the image of $\sigma_{i}^{\prime} \sigma_{j}: C^{0} \rightarrow C^{2}$ by $G_{i j}$, and the image of $\sigma_{i}^{\prime} \pi_{0}: C^{1} \rightarrow C^{2}$ by $G_{i}$. Then, by $(1.8 .13)^{\prime}$, we have $G_{i j}=G_{j i}$ and $G_{i i}=0$.

LEMma 1.8.1. We have

(i) if $i \neq j$, the maps

$$
\sigma_{i}^{\prime} \sigma_{j}: C^{0} \rightarrow G_{i j}, \quad \sigma_{i}^{\prime}: E_{0} \rightarrow G_{i}
$$

are isomorphisms;

(ii) the sum

$$
\sum_{1 \leqq i<j \leqq k} G_{i j}+\sum_{1 \leqq i \leqq k} G_{i}
$$

is direct. 
Proof. To prove (i), we determine the kernel of $\sigma_{i}^{\prime}$. By Quillen's theorem (Theorem 1.7.2), the sequence

$$
0 \longrightarrow C^{0} \stackrel{\sigma_{i}}{\longrightarrow} C^{1} \stackrel{\sigma_{i}{ }^{\prime}}{\longrightarrow} C^{2}
$$

is exact since $d x^{i}$ is noncharacteristic. Thus, $\operatorname{ker} \sigma_{i}^{\prime}=\operatorname{im} \sigma_{i}=E_{i}$. Since $E_{0}$ and $E_{j}$ are disjoint from $E_{i}$ for $i \neq j$, we infer that $\sigma_{i}^{\prime}: E_{0} \rightarrow G_{i}$ is an isomorphism. It is immediate that $\sigma_{i}^{\prime} \sigma_{j}: C^{0} \rightarrow G_{i j}$, where $i \neq j$, is an isomorphism.

Now suppose that there exist $g_{i} \in G_{i}$ and $g_{i j} \in G_{i j}$ such that

$$
\sum_{1 \leqq i<j \leqq 1}^{k} g_{i j}+\sum_{i=1}^{k} g_{i}=0 .
$$

By part (i) and (1.8.13) there exist $u_{i j} \in C^{0}, u_{i j}=-u_{j i}$, and $u_{i} \in E_{0}$ such that $g_{i j}=\sigma_{i}^{\prime} \sigma_{j}\left(u_{i j}\right)$ and $g_{i}=\sigma_{i}^{\prime}\left(u_{i}\right)$, and

$$
\sum_{i, j=1}^{k} \sigma_{i}^{\prime} \sigma_{j}\left(u_{i j}\right)+\sum_{i=1}^{k} \sigma_{i}^{\prime}\left(u_{i}\right)=0 .
$$

It is sufficient to show that $u_{i}=0$ and $u_{i j}=0$. The relation (1.8.15) is the same as

$$
\sigma\left(D^{\prime}\right)\left(\sum_{i, j=1}^{k} d x^{i} \otimes\left(\sigma_{j}\left(u_{i j}\right)+u_{i}\right)\right)=0 .
$$

By the exactness of (1.8.4) we have

$$
\sum_{i, j=1}^{k} d x^{i} \otimes\left(\sigma_{j}\left(u_{i j}\right)+u_{i}\right)=\sigma(D)\left(\sum_{l, m} d x^{l} \otimes d x^{m} \otimes u_{l m}^{\prime}\right)
$$

where $u_{l m}^{\prime} \in C^{0}$ and $u_{l m}^{\prime}=u_{m l}^{\prime}$. Thus

$$
\sum_{i, j=1}^{k} d x^{i} \otimes\left(\sigma_{j}\left(u_{i j}\right)+u_{i}\right)=\sum_{l, m=1}^{k} d x^{l} \otimes \sigma_{m}\left(u_{l m}^{\prime}\right)
$$

and it follows that

$$
\sum_{j=1}^{k} \sigma_{j}\left(u_{i j}\right)+u_{i}=\sum_{m=1}^{k} \sigma_{m}\left(u_{i m}^{\prime}\right) .
$$

Since $E_{0}$ and $E_{j}$ are disjoint from $E_{m}$ for $j \neq m$, we conclude that $u_{i}=0$ and $\sigma_{j}\left(u_{i j}\right)=\sigma_{j}\left(u_{i j}^{\prime}\right)$. Since $\sigma_{j}$ is injective, we infer that $u_{i j}=u_{i j}^{\prime}$. Since $u_{i j}$ is skew-symmetric and $u_{i j}^{\prime}$ symmetric, we have $u_{i j}=u_{i j}^{\prime}=0$ and this proves (ii). 
Let

$$
\pi_{i j}^{\prime}: C^{2} \rightarrow G_{i j}, \quad \pi_{i}^{\prime}: C^{2} \rightarrow G_{i}
$$

be the projections, and set

$$
\begin{aligned}
& D_{i j}^{\prime}=-\left(\sigma_{i}^{\prime} \sigma_{j}\right)^{-1} \pi_{i j}^{\prime} D^{\prime}, \\
& D_{i}^{\prime}=\left(\sigma_{i}^{\prime}\right)^{-1} \pi_{i}^{\prime} D^{\prime} .
\end{aligned}
$$

Proposition 1.8.1. We have

$$
\begin{aligned}
& {\left[D_{i}, D_{j}\right]=D_{i j}^{\prime} D_{0},} \\
& D_{0} D_{i}=D_{i}^{\prime} D_{0},
\end{aligned}
$$

where $\left[D_{i}, D_{j}\right]=D_{i} D_{j}-D_{j} D_{i}$. In particular, $D_{i}$ maps ker $D_{0}$ into $\operatorname{ker} D_{0}$ and $D_{i} D_{j}=D_{j} D_{i}$ on $\operatorname{ker} D_{0}$.

Proof. By (1.8.14) we have

$$
\begin{aligned}
D_{i j}^{\prime} D_{0} & =-\left(\sigma_{i}^{\prime} \sigma_{j}\right)^{-1} \pi_{i j}^{\prime} D^{\prime} D_{0} \\
& =\left(\sigma_{i}^{\prime} \sigma_{j}\right)^{-1}\left\{\pi_{i j}^{\prime}\left(\sum_{l<m} \sigma_{l}^{\prime} \sigma_{m}\left(D_{l} D_{m}-D_{m} D_{l}\right)\right)\right\} \\
& =D_{i} D_{j}-D_{j} D_{i}=\left[D_{i}, D_{j}\right] .
\end{aligned}
$$

Similarly we have, again by (1.8.14),

$$
D_{i}^{\prime} D_{0}=\left(\sigma_{i}^{\prime}\right)^{-1} \pi_{i}^{\prime} D^{\prime} D_{0}=\left(\sigma_{i}^{\prime}\right)^{-1} \pi_{i}^{\prime}\left(\sum_{l=1}^{k} \sigma_{l}^{\prime} \pi_{0} D D_{l}\right)=D_{0} D_{i},
$$

and this is the second formula (1.8.17).

The commutation relations of Proposition 1.8.1 yield commutation relations for the rorresponding symbols. Let $\eta$ be an arbitrary covector. Then

$$
\begin{aligned}
& {\left[\sigma_{\eta}\left(D_{i}\right), \sigma_{\eta}\left(D_{j}\right)\right]=\sigma_{\eta}\left(D_{i j}^{\prime}\right) \sigma_{\eta}\left(D_{0}\right),} \\
& \sigma_{\eta}\left(D_{0}\right) \sigma_{\eta}\left(D_{i}\right)=\sigma_{\eta}\left(D_{i}^{\prime}\right) \sigma_{\eta}\left(D_{0}\right) .
\end{aligned}
$$

Hence $\sigma_{\eta}\left(D_{i}\right)$ maps $\operatorname{ker} \sigma_{\eta}\left(D_{0}\right)$ into $\operatorname{ker} \sigma_{\eta}\left(D_{0}\right)$, and $\sigma_{\eta}\left(D_{i}\right) \sigma_{\eta}\left(D_{j}\right)$ $=\sigma_{\eta}\left(D_{j}\right) \sigma_{\eta}\left(D_{i}\right)$ on $\operatorname{ker} \sigma_{\eta}\left(D_{0}\right)$. We remark that these relations are trivial if $\eta \in U$ since they then follow from (1.8.8).

We conclude this section by outlining briefly Guillemin's program for the solution of a local overdetermined Cauchy problem based on the preceding formulas.

Let a local coordinate $\left(x^{1}, \cdots, x^{k}\right)$ be chosen in the manner de- 
scribed above such that, at each point of the surface $S$ defined by $x^{1}=0, x^{2}=0, \cdots, x^{k}=0$, the differentials $d x^{1}, d x^{2}, \cdots, d x^{k}$ span a maximal noncharacteristic subspace of the cotangent space at that point. The Cauchy datum is a section $f_{0}$ of $C^{0}$ over $S$ satisfying the equation

$$
D_{0} f_{0}=0 .
$$

The problem is to extend $f_{0}$ to a section $f$ of $C^{0}$ defined over a neighborhood in $X$ of each interior point of $S$ and satisfying there the equation $D f=0$. It is natural to try to construct $f$ by the following inductive procedure. Let $S_{0}=S$, let $S_{1}$ be the surface defined by $x^{1}=0, \cdots x^{k-1}$ $=0, S_{2}$ the surface defined by $x^{1}=0, \cdots, x^{k-2}=0$ and finally let $S_{k}$ be a neighborhood suitable for the definition of the desired solution $f$. We are given $f_{0}$ on $S_{0}$ satisfying (1.8.19) and we try to find $f_{1}$ (section of $C^{0}$ ) on $S_{1}$ such that $D_{1} f_{1}=D_{0} f_{1}=0$ and $f_{1}=f_{0}$ on $S_{0}$. We assume that we can solve the following Cauchy problem: $D_{1} f_{1}=0$ on $S_{1}, f_{1}=f_{0}$ on $S_{0}$. Then $D_{0} D_{1} f_{1}=0$ on $S_{1}$, and it follows from the second formula (1.8.17) that $D_{1}^{\prime} D_{0} f_{1}=0$ on $S_{1}$. Thus $g=D_{0} f_{1}$ is a solution of the Cauchy problem $D_{1}^{\prime} g=0$ on $S_{1}, g=0$ on $S_{0}$. We assume the uniqueness of the solution of the Cauchy problem for $D_{1}^{\prime}$ and then we have $D_{0} f_{1}=0$ as desired. Next, we find $f_{2}$ (section of $C^{0}$ ) on $S_{2}$ satisfying $D_{2} f_{2}=D_{1} f_{2}=D_{0} f_{2}=0$ on $S_{2}$ and $f_{2}=f_{1}$ on $S_{1}$, and we assume that we can solve uniquely the Cauchy problem $D_{2} f_{2}=0$ on $S_{2}$ and $f_{2}=f_{1}$ on $S_{1}$. Then $D_{0} D_{2} f_{2}=0$ on $S_{2}$ and therefore, by the second formula (1.8.17), $D_{2}^{\prime} D_{0} f_{2}=0$ on $S_{2}$ and $D_{0} f_{2}=D_{0} f_{1}=0$ on $S_{1}$. Assuming the uniqueness of the solution of the Cauchy problem for $D_{2}^{\prime}$, we have $D_{0} f_{2}=0$ on $S_{2}$. Since $D_{0} f_{2}=0$ we have, by the first formula (1.8.17), $D_{2} D_{1} f_{2}=D_{1} D_{2} f_{2}$ $=0$ on $S_{2}$, and we also have $D_{1} f_{2}=D_{1} f_{1}=0$ on $S_{1}$. Hence, by the uniqueness of the Cauchy problem for $D_{2}$, we infer that $D_{1} f_{2}=0$ on $S_{2}$. Continuing in this fashion, we arrive at a section $f_{k}$ of $C^{0}$ on $S_{k}$ satisfying $D_{0} f_{k}=D_{1} f_{k}=\cdots=D_{k} f_{k}=0, f_{k}=f_{0}$ on $S_{0}$, and it follows from (1.8.7) that

$$
D f_{k}=D_{0} f_{k}+\sum_{i=1}^{k} \sigma_{d x i}(D) D_{i} f_{k}=0 .
$$

The desired solution is therefore obtained by taking $f=f_{k}$.

The solvability by this procedure of the Cauchy problem for $D$ requires the following theorems: (i) existence and uniqueness of solutions of the Cauchy problems for the operators $D_{i}, 1 \leqq i \leqq k$; (ii) uniqueness of solutions of the Cauchy problems for $D_{i}^{\prime}, 1 \leqq i \leqq k$. These theorems are not yet completely established. 


\section{Existence theorems.}

2.1. Analytic operators. Suppose that the category is that of real analytic manifolds and maps. In particular, the manifold $X$ and all bundles over it are real analytic. Let $D: E \rightarrow F$ be a (real) analytic operator from $E$ to $F$, i.e., an operator which sends analytic sections of $E$ into analytic sections of $F$. It is easily seen that the theorems of Chapter 1 hold in the analytic sense. In particular, suppose that the operator is formally integrable. Then (see Proposition 1.6.1 and Theorem 1.6.2) there exist an analytic vector bundle $G_{1}$ and analytic operator $D_{1}: F \rightarrow G_{1}$ such that the complex of sheaves

$$
E \stackrel{D}{\longrightarrow} F \stackrel{D_{1}}{\longrightarrow} G_{1}
$$

is exact if and only if the first or second sequence attached to the operator (each analytic) is exact at degree 1 in the stable range. We have

THEOREM 2.1.1. In the case of a real analytic, formally integrable operator, the complex (2.1.1) is exact.

This theorem follows, by Proposition 1.6.1 and Theorem 1.6.2, from the following proposition:

PROPOSITION 2.1.1. In the real analytic case, the first (therefore also the second) complex attached to a formally integrable operator is exact in the stable range.

The proposition is an easy consequence of the formal theory developed in the preceding chapter. However, because it may have independent interest, we shall sketch a proof using the homotopy operator of Buttin [2] which was mentioned briefly at the end of $\$ 1.3$.

We obtain from the first complex attached to the differential operator, by passage to the projective limit, the complex (compare (1.3.9))

$$
0 \rightarrow \Theta \stackrel{j_{\infty}}{\rightarrow} R_{\infty} \stackrel{D}{\rightarrow} T^{*} \otimes R_{\infty} \stackrel{D}{\rightarrow} \cdots \stackrel{D}{\rightarrow} \Lambda^{n} T^{*} \otimes R_{\infty} \rightarrow 0
$$

where $R_{\infty}$ is the projective limit of the $R_{m}$. For this sequence Buttin (loc. cit.) defines a formal local homotopy operator $K$ which is obtained, in the manner described in $\$ 1.3$, from the usual local homotopy operator $k$ for the exterior differential operator $d$ on differential forms. We shall now describe the operator $K$.

Let $U$ be an open set covered by a local (real) analytic coordinate $x=\left(x^{1}, x^{2}, \cdots, x^{n}\right)$, and suppose that $U$ is starlike with respect to the center $x=0$ of this coordinate. We have the usual homotopy operator 
$k$ for the forms $\sigma$ on $U$ satisfying

$$
\begin{aligned}
& k d \sigma+d k \sigma=\sigma, \operatorname{deg} \sigma>0, \\
& k d \sigma=\sigma(x)-\sigma(0), \quad \operatorname{deg} \sigma=0 .
\end{aligned}
$$

In fact, let the differential form $\sigma$ of degree $r$, expressed in terms of the local coordinate, have the form

$$
\sigma=\sum \sigma_{j_{1} \cdots j_{r}}(x) d x^{j_{1}} \wedge \cdots \wedge d x^{j_{r}}
$$

where the summation is over $j_{1}, \cdots, j_{r}$ satisfying $j_{1}<\cdots<j_{r}$. We regard $x=\left(x^{1}, x^{2}, \cdots, x^{n}\right)$ as a local vector field

$$
x=\sum_{i=1}^{n} x^{i} \frac{\partial}{\partial x^{i}}
$$

which operates by contraction on $\sigma$ as follows:

$$
\begin{aligned}
& \sigma \pi x=\sum \sigma_{j_{1}} \ldots j_{r} \\
&\left\{\sum_{i=1}^{r}(-1)^{i-1} x^{j_{i}} d x^{j_{1}} \wedge \cdots\right. \\
&\left.\wedge d x^{j_{i-1}} \wedge d x^{j_{i+1}} \wedge \cdots d x^{j_{r}}\right\}, \operatorname{deg} \sigma>0, \\
&=0, \quad \operatorname{deg} \sigma=0 .
\end{aligned}
$$

Let

$$
V \sigma=d \sigma \pi x+d(\sigma \pi x)
$$

denote the Lie derivative of $\sigma$ along the vector field $x$, where

$$
V \sigma=\left\{\sum_{j=1}^{n} x^{j} \frac{\partial}{\partial x^{j}} \sigma_{j_{1}} \ldots j_{r}+r \sigma_{j_{1}} \ldots j_{r}\right\} d x^{j 1} \wedge \cdots \wedge d x^{j_{r}} .
$$

Then $V$ is invertible for $r \geqq 1$, that is the equation

$$
\sum_{j=1}^{n} x^{j} \frac{\partial f}{\partial x^{j}}+r f=g
$$

where $g$ is a differentiable function, has a unique differentiable solution $f=V^{-1} g$ given by

$$
f=\int_{0}^{1} t^{r-1} g(t x) d t
$$

for $r \geqq 1$, where $t x=\left(t x^{1}, \cdots, t x^{n}\right)$. Then 


$$
\begin{aligned}
k \sigma & =V^{-1} \sigma \pi x, \quad \operatorname{deg} \sigma>0, \\
& =0, \quad \operatorname{deg} \sigma=0 .
\end{aligned}
$$

A straight-forward generalization of this process yields a formal homotopy operator $K$ for sections $\sigma$ of $\Lambda^{r} T^{*} \otimes R_{\infty}$ over $U$. We define $W \sigma=k D \sigma+D k \sigma$ where

$$
\begin{aligned}
W \sigma & =\sigma-(k \delta+\delta k), \quad \operatorname{deg} \sigma>0, \\
& =\sigma(x)-\sigma(0)-k \delta \sigma, \quad \operatorname{deg} \sigma=0,
\end{aligned}
$$

and $\delta$ is the local formal differential operator which, expressed in terms of the local coordinates, has the form (compare \$1.3)

$$
(\delta \sigma)_{\alpha}=\sum d x^{i} \wedge\left(\delta_{i} \sigma\right)_{\alpha}
$$

where $\left(\delta_{i} \sigma\right)_{\alpha}=\sigma_{\alpha+1_{i}}$. In general, $W$ is not invertible but it is invertible if the series $\sum_{i=0}^{\infty}(\delta k)^{l}$ converges. We define formally

$$
\begin{aligned}
K \sigma & =k \sum_{l=0}^{\infty}(\delta k)^{l} \sigma, \quad \operatorname{deg} \sigma>0, \\
& =0, \quad \operatorname{deg} \sigma=0 .
\end{aligned}
$$

Then in the formal sense we have, in analogy with (2.1.3),

$$
\begin{aligned}
& K D \sigma+D K \sigma=\sigma, \quad \operatorname{deg} \sigma>0, \\
& K D \sigma=\sigma(x)-\sum_{l=0}^{\infty}(k \delta)^{l} \sigma(0), \quad \operatorname{deg} \sigma=0 .
\end{aligned}
$$

We remark that, among formal homotopy operators on sections of $\wedge T^{*} \otimes R_{\infty}$, the above operator $K$ is uniquely determined by the following pair of conditions:

(i) $K D+D K=$ identity, $\quad \operatorname{deg}>0$,

(ii) $K \sigma$ is in the image of $\Lambda_{x}$; , i.e., $K \sigma=\omega \pi x$.

The explicit form of $K$ is given by the formula

$$
(K \sigma)_{\alpha}=\left(\int_{0}^{1} t^{r-1}\left(\sum_{l=0}^{\infty}(1-t)^{\iota}\left(\sum_{|\beta|=l} \frac{x^{\beta}}{\beta !} \sigma_{\alpha+\beta}(t x)\right)\right) d t\right) \pi x .
$$

In terms of local coordinates, an element $\sigma$ of $\Lambda^{r} T^{*} \otimes R_{\infty}$ has the components $\sigma_{\alpha}^{j}$, where the superscript $j$ refers to a fibre coordinate for $E$, and

$$
\sigma_{\alpha}^{j}=\sum \sigma_{\alpha_{r} j_{1} \cdots j_{r}}^{j} d x^{j_{1}} \wedge \cdots \wedge d x^{j_{r}}
$$


Let

$$
\|\sigma\|_{m}=\sup \left|\sigma_{\alpha_{, j} j_{1} \cdots j_{r}}^{j}(x)\right|
$$

for $x \in U$, all $j, j_{1}, \cdots, j_{r}$ and all $\alpha$ satisfying $|\alpha|=\alpha_{1}+\cdots+\alpha_{n}$ $=m$. We shall say that $\sigma$ satisfies condition (C) on $U$ if and only if there exist numbers $M$ and $h$ such that

$$
\|\sigma\|_{m} \leqq M m ! h^{m}
$$

for all $m$. Condition (C) means that the $\sigma_{\alpha}$ behave like the derivatives of an $E$-valued analytic function. We have (Buttin [2]):

Proposition 2.1.2. Let $\sigma$ be a section of $\wedge^{r} T^{*} \otimes R_{\infty}$ which satisfies the condition (C) on some disc. Then $K \sigma$ exists and is a local section of $\Lambda^{r-1} T^{*} \otimes R_{\infty}$

From this proposition we obtain Proposition 2.1.1. In fact, let $\sigma_{m}$ be a local analytic section of $\Lambda^{r} T^{*} \otimes R_{m}$, where $r \geqq 1$ and $m \geqq \mu$ (integer of Theorem 1.4.1), and suppose that $D \sigma_{m}=0$. Since $m \geqq \mu$, the element $\sigma_{m}$ can be lifted up to a local analytic section $\sigma$ of $\Lambda^{r} T^{*} \otimes R_{\infty}$ satisfying $D \sigma=0$ and also condition (C) on a small disc. The proof that condition (C) is satisfied is based on an estimate in the $\delta$-Poincare lemma, namely, if $\delta \sigma=0$, the determination of an element $\tau$ satisfying $\delta \tau=\sigma$ which is suitably bounded in terms of $\sigma$ (see Ehrenpreis, Guillemin and Sternberg [5], Sweeney [23(a)], and Goldschmidt $[6(e)])$. By Proposition 2.1.2 and the first formula (2.1.4), we have $\sigma=D \tau$ where $\tau=K \sigma$. By projection, $\tau$ yields a local section $\tau_{m+1}$ of $\Lambda^{r-1} T^{*} \otimes R_{m+1}$ satisfying $D \tau_{m+1}=\sigma_{m}$. This proves Proposition 2.1.1 and hence Theorem 2.1.1.

We conclude this section with a theorem about elliptic operators of analytic type.

Definition 2.1.1. We say that an operator $D: E \rightarrow F$ is of analytic type if it is formally integrable and if, to each point of $X$, there is a neighborhood of the point on which a local coordinate $\left(x^{1}, x^{2}, \cdots\right.$, $x^{n}$ ) of $X$ and local bundle coordinates for $E, F$ can be chosen such that the operator, expressed in terms of these coordinates, is real analytic.

We have

Theorem 2.1.2. Suppose that $D: E \rightarrow F$ is an elliptic operator of analytic type. Then the complex (2.1.1), where the terms are germs of differentiable sections, is exact.

By Theorem 1.6.2, this theorem is a consequence of the following proposition: 
Proposition 2.1.3. Suppose that $D: E \rightarrow F$ is an elliptic operator of analytic type. Then the second complex attached to the operator, whose terms are germs of differentiable sections, is exact (in the stable range).

Suppose that the hypothesis of Proposition 2.1.3 is satisfied. Let $C^{i}=C_{m+1}^{i}$ be defined by (1.6.3) where $m \geqq \mu$ (integer of Theorem 1.4.1). For simplicity we drop the superscript on the operators of the second complex attached to the given operator and write the complex in the form (1.6.20).

Let $x_{0}$ be an arbitrary point of $X$. Since the operator from $E$ to $F$ is of analytic type, we can choose a local coordinate on a neighborhood $U$ of $x_{0}$, and coordinates for the bundles restricted to $U$, such that the operator is real analytic. Next, we choose a real analytic riemannian metric on $U$ and a real analytic metric along the fibres of the vector bundle $J_{m}(E)$ restricted to $U$. Then, for each $i$, real analytic metrics are induced along the fibres of $\Lambda^{i} T^{*} \otimes J_{m}(E)$ and hence along the fibres of each of the sub-bundles $\Lambda^{i} T^{*} \otimes R_{m}, \Lambda^{i} T^{*} \otimes g_{m}$. Finally choose a local real analytic splitting (1.6.17) (with $m$ replaced by $m+1)$; then we have the isomorphism

$$
C^{i}=C_{m+1}^{i} \cong\left(\Lambda^{i} T^{*} \otimes R_{m}\right) \oplus \delta\left(\Lambda^{i} T^{*} \otimes g_{m+1}\right) .
$$

Hence we obtain, for each $i$, a local analytic metric along the fibres of $C^{i}$.

Since the operator from $E$ to $F$ is elliptic, it follows from Theorem 1.7.1 that the laplacian $\square=D D^{*}+D^{*} D: C^{i} \rightarrow C^{i}$, where $D$ denotes the operator of the second sequence and $D^{*}$ its formal adjoint defined in terms of the real analytic metrics, is a strongly elliptic operator of order 2 . And $\square$ is clearly an analytic operator. By standard theorems concerning elliptic operators, there exists a Green's operator $G$ on $U$ (assumed to be a sufficiently small neighborhood) such that $\square G=I$ (identity). We observe that

$$
\square(G D-D G)=0 .
$$

Now suppose that $\tilde{u}$ is a germ of differentiable section of $C^{i}, i \geqq 1$, at the point $x_{0}$, where $\tilde{u}$ satisfies $D \tilde{u}=0$. Then $u$ is represented by a section $u$ of $C^{i}$ over a neighborhood of $x_{0}$, which we may assume is $U$, and $D u=0$. Let $V \subset U$ be a neighborhood of $x_{0}$ whose closure is a compact subset of $U$, and let $\rho$ be a differentiable function whose support is a compact subset of $U$ and suppose that $\rho=1$ on $V$. We set $v=\rho u$.

We have

$$
v=\square G v=D D^{*} G v+D^{*} D G v=D\left(D^{*} G v\right)+D^{*} G D v+H v
$$


where, by (2.1.5), $\square H v=0$. Moreover, we have

$$
\square\left(D^{*} G D v\right)=D^{*} D v=0
$$

on $V$, since $D v=0$ there. Since $\square$ is a strongly elliptic, analytic operator of second order, it follows from known theorems that $D^{*} G D v$ and $H v$ are real analytic on $V$. Setting $w=D^{*} G D v+H v$, we thus have $v=D\left(D^{*} G v\right)+w$ where $w$ is real analytic on $V$ and $D w=0$. It follows from Proposition 2.1.1 that $w=D w^{\prime}$ on some possibly smaller neighborhood of $x_{0}$ and this proves Proposition 2.1.3.

It is a consequence of Proposition 2.1.3, and the fact that any elliptic transitive continuous pseudogroup is locally isomorphic to an analytic elliptic (transitive continuous) pseudogroup, that the second complex associated with the linear equations defining its infinitesimal transformations is exact.

2.2. The $\delta$-estimate. We return to the differentiable $\left(C^{\infty}\right)$ category, and we suppose that $D: E \rightarrow F$ is a formally integrable operator of order $k$. We shall describe an inequality (estimate) for the $\delta$-operator of $\$ 1.4$ which, if the given operator from $E$ to $F$ satisfies it, implies interesting properties of the operator. The results of this section are due to B. MacKichan [16]. We shall omit almost all proofs, for which we refer the reader to MacKichan, loc. cit.

Let

$$
0 \longrightarrow \Theta \longrightarrow C^{0} \stackrel{D^{0}}{\longrightarrow} C^{1} \stackrel{D^{1}}{\longrightarrow} \ldots \stackrel{D^{n-1}}{\longrightarrow} C^{n} \longrightarrow 0
$$

be the second complex (1.6.6) associated with the operator from $E$ to $F$, where $C^{i}=C_{m}^{i}$ is defined by (1.6.3) and $m \geqq \mu$ (integer of Theorem 1.4.1). We have the symbol maps

$$
\sigma_{i}\left(D^{l}\right): S^{i+1} T^{*} \otimes C^{l} \rightarrow S^{i} T^{*} \otimes C^{l+1},
$$

and we denote the kernel of $\sigma_{i}\left(D^{l}\right)$ by $g_{i+1}^{l}$. We remark that $g_{i+1}^{0}$ is isomorphic to $g_{m+i+1}$.

Assume that riemannian metrics have been chosen along the fibres of the cotangent bundle $T^{*}$ of $X$ and the other vector bundles involved.

Definition 2.2.1. We say that the operator $D: E \rightarrow F$ satisfies the $\delta$-estimate if and only if, on the sequence

$$
0 \rightarrow \stackrel{0}{0} \stackrel{\delta}{\rightarrow} T^{*} \otimes \stackrel{0}{0} \stackrel{\delta}{\rightarrow} \Lambda^{2} T^{*} \otimes C^{0},
$$

we have the inequality $\|\delta \chi\|^{2} \geqq \frac{1}{2}\|\chi\|^{2}$ for all $\chi \in\left(T^{*} \otimes g_{1}^{0}\right) \cap \operatorname{ker} \delta^{*}$, where $\delta^{*}$ is the adjoint of $\delta$ (defined in terms of the metric). 
We have

Proposition 2.2.1. The operator $D: E \rightarrow F$ satisfies the $\delta$-estimate if and only if

$$
\|\delta x\|^{2} \geqq \frac{1}{2}(m+1)^{2}\|x\|^{2}
$$

for all $\chi \in\left(T^{*} \otimes g_{m+1}\right) \cap \operatorname{ker} \delta^{*}$. Moreover, if $D$ satisfies the $\delta$-estimate then, for $i, l \geqq 1$, we have $\|\delta \chi\|^{2} \geqq\left(l^{2} / i\right)\|\chi\|^{2}$ for $\chi \in\left(\bigwedge^{i-1} T^{*} \otimes g_{l}^{0}\right) \cap \operatorname{ker} \delta^{*}$.

Here and elsewhere $\delta^{*}$ denotes the adjoint of the operator indicated by the assertion.

As an immediate corollary of Proposition 2.2.1, we see that the sequences

$$
0 \rightarrow g_{l}^{0} \stackrel{\delta}{\rightarrow} T^{*} \otimes g_{l-1}^{0} \stackrel{\delta}{\rightarrow} \cdots \stackrel{\delta}{\rightarrow} \Lambda^{l-1} T^{*} \otimes g_{1}^{0} \rightarrow \Lambda^{l} T^{*} \otimes C^{0}
$$

are exact, and hence $g_{1}$ is involutive (see Definition 1.4.1) or equivalently $g_{m+1}$ is involutive.

The following result should also be noted:

Proposition 2.2.2. If D satisfies the $\delta$-estimate, then $\|\delta \chi\|^{2} \geqq \frac{1}{2}\|\chi\|^{2}$ for all $\chi \in\left(T^{*} \otimes g_{1}^{l}\right) \cap \operatorname{ker} \delta^{*}$.

The next proposition shows that the constant $\frac{1}{2}$ occurring in the $\delta$-estimate is distinguished.

Proposition 2.2.3. (i) If

$$
\|\delta x\|^{2} \geqq c(1,1)\|x\|^{2}
$$

for $\chi \in\left(T^{*} \otimes g_{1}^{0}\right) \cap \operatorname{ker} \delta^{*}$, then

$$
\|\delta x\|^{2} \geqq c(1, l)\|x\|^{2}
$$

for $\chi \in\left(T^{*} \otimes g_{\imath}^{0}\right) \cap \operatorname{ker} \delta^{*}$, where

$$
c(1, l)=\left\{1-(l-1)^{2} / 4 c(1, l-1\} l^{2} .\right.
$$

In particular, if $c(1,1)=\frac{1}{2}$, then $c(1, l)=l^{2} / 2$.

(ii) If

$$
\|\delta x\|^{2} \geqq c(1, l)\|x\|^{2}
$$

for $\chi \in\left(T^{*} \otimes g_{\imath}^{0}\right) \cap \operatorname{ker} \delta^{*}$, then

$$
\|\delta x\|^{2} \geqq c(i, l)\|x\|^{2}
$$

for $\chi \in\left(\Lambda^{i} T^{*} \otimes g_{l}^{0}\right) \cap \operatorname{ker} \delta^{*}$, where

$$
c(i, l)=\frac{i^{2} c(i-1, l)-l^{2}}{(i+1)(i-1)} .
$$


In particular, if $c(1, l)=l^{2} / 2$, then $c(\mathrm{i}, l)=l^{2} /(\mathrm{i}+1)$.

From (i) of Proposition 2.2.3 we see that the constant $\frac{1}{2}$ in the $\delta$-estimate is the least constant which insures that $c(1, l)>0$ for all $l$, and from (ii) that $\frac{1}{2}$ is the least value for $c(1,1)$ such that $c(i, l)>0$ for all $i$ and $l$.

The following proposition is due to V. W. Guillemin:

Proposition 2.2.4. If $D$ satisfies the $\delta$-estimate and if $H$ is a subspace of $T^{*}$, then $g_{1}^{0} \cap\left(H \otimes C^{0}\right)$ also satisfies the $\delta$-estimate.

The proof of the following theorem, due to MacKichan (loc. cit.), depends on Proposition 2.2.4.

Theorem 2.2.1. Suppose that $D$ satisfies the $\delta$-estimate. Let $U$ be a maximal noncharacteristic subspace of $T^{*}$, and let $D_{0}$ and $D_{i}, 1 \leqq i \leqq k$, be the local differential operators constructed in terms of $U$ in $\$ 1.8$. Then, if $\eta$ is a covector not belonging to $U$ we have, on $\operatorname{ker} \sigma_{\eta}\left(D_{0}\right)$, the commutation relations

$$
\sigma_{\eta}\left(D_{i}\right) \sigma_{\eta}\left(D_{j}^{*}\right)=\sigma_{\eta}\left(D_{j}^{*}\right) \sigma_{\eta}\left(D_{i}\right), \quad 1 \leqq i, j \leqq k,
$$

where $\sigma_{\eta}\left(D_{j}^{*}\right)=\sigma_{\eta}\left(D_{j}\right) *$ denotes the adjoint of $\sigma_{\eta}\left(D_{j}\right), D_{j}^{*}$ the adjoint of $D_{j}$.

We shall not prove Theorem 2.2.1, but shall only describe briefly how the $\delta$-estimate enters into the proof. We suppose that $\eta$ is a covector not belonging to the maximal noncharacteristic subspace $U$ in terms of which $D_{0}$ and $D_{i}$ are defined and we write, for simplicity,

$$
A_{i}=\sigma_{\eta}\left(D_{i}\right), \quad i=1,2, \cdots, k \text {. }
$$

Moreover, let $A_{0}$ be the identity map of $C^{0}$ and set $B=\sum_{i=0}^{k} A_{i}^{*} A_{i}$. It is obvious that $B$ is selfadjoint and positive-definite (since $A_{0}$ is the identity), and $B$ is an automorphism of $\operatorname{ker} \sigma_{\eta}\left(D_{0}\right)$. A calculation shows that, if

$$
\chi=\sum_{i, j=0}^{k} \xi^{j} \otimes \xi^{i} \otimes A_{i} c_{j}
$$

where $\xi^{0}=\eta, \xi^{i}=d x^{i}$ for $i=1,2, \cdots, k$ (basis of $U$ ), and $c_{j} \in$ ker $\sigma_{\eta}\left(D_{0}\right)$, then $\delta^{*} \chi=0$ if and only if

$$
\sum_{j=0}^{k} A_{j}^{*} B c_{j}=0
$$

Moreover,

$$
\|\delta x\|^{2}-\frac{1}{2}\|x\|^{2}=-\sum_{i, j=0}^{k}\left\langle A_{j} c_{i}, A_{i} c_{j}\right\rangle
$$


where $\langle\cdots\rangle$ denotes the scalar product defined in terms of the metrics. Then the $\delta$-estimate is equivalent to the assertion that $\delta^{*} \chi=0$ implies

$$
\sum_{i, j=0}^{k}\left\langle A_{j} c_{i}, A_{i} c_{j}\right\rangle \leqq 0 .
$$

From (2.2.2) the desired commutation relations of Theorem 2.2.1, namely $A_{i} A_{j}^{*}=A_{j}^{*} A_{i}$ on ker $\sigma_{\eta}\left(D_{0}\right)$, are obtained by a calculation (which we omit).

The point of exhibiting the inequality (2.2.2) is that the $\delta$-estimate seems to imply inequalities of this type. An inequality of similar form will be derived from the $\delta$-estimate in the next section.

2.3. The Neumann problem. Let $X$ be a smooth riemannian manifold of dimension $n$, and let $\Omega \subset X$ be a compact, $n$-dimensional manifold whose boundary is imbedded differentiably in $X$ as a compact $(n-1)$-dimensional submanifold. The metric on $X$ induces metrics on $\Omega$ and on its boundary $\omega=\partial \Omega$. Let $E$ and $F$ be vector bundles over $X$, and let a formally integrable elliptic differential operator $D: E \rightarrow F$ be given. We suppose that $m$ is a fixed integer which is greater than or equal to the integer $\mu$ occurring in Theorem 1.4.1, and we let $C^{i}=C_{m+1}^{i}$ be defined by (1.6.3) in terms of the differential operator. For simplicity we drop the superscripts on the differential operators $D^{i}$ connecting the terms (sheaves) in the second sequence and write it in the form (1.6.20). Confusion between these operators and the given operator from $E$ to $F$ will not occur because the latter seldom enters the discussion and, when it enters, the bundles $E, F$ are also exhibited. Next, we choose an inner product along the fibres of the bundle $J_{m}(E) \rightarrow X$ which, together with the riemannian metric, induces, for each $i$, inner products along the fibres of the bundles $\Lambda^{i} T^{*} \otimes J_{m}(E)$ and hence along the fibres of each of the sub-bundles $\Lambda^{i} T^{*} \otimes R_{m}$, $\Lambda^{i} T^{*} \otimes g_{m}$ and $\delta\left(\Lambda^{i} T^{*} \otimes g_{m+1}\right)$. Finally choose a splitting (1.6.17); then we have the isomorphism

$$
C^{i}=C_{m+1}^{i} \cong\left(\Lambda^{i} T^{*} \otimes R_{m}\right) \oplus \delta\left(\Lambda^{i} T^{*} \otimes g_{m+1}\right) .
$$

Hence we obtain, for each $i$, an inner product along the fibres of $C^{i}$.

We denote by $\Gamma\left(\Omega, C^{i}\right)$ the space of all differentiable sections of $C^{i}$ over $\Omega$; these sections are smooth up to and including the boundary $\omega$ of $\Omega$ and can be extended smoothly across $\omega$. If $u, v \in \Gamma\left(\Omega, C^{i}\right)$ we have the inner product

$$
\langle u, v\rangle=\int_{\Omega}\langle u, v\rangle_{x} d v
$$


where $d v$ is the riemannian volume element and $\langle\cdots\rangle_{x}$ is the inner product along the fibre of $C^{i}$ over the point $x \in X$. We write $\|u\|$ $=\langle u, u\rangle^{\frac{1}{2}}$ for the corresponding norm. It is convenient in practice to complexify all bundles which occur in order to introduce the required Fourier transform spaces, and then the inner products are hermitian. However, this complexification is not necessary for our purpose here. We denote by $L_{2}\left(\Omega, C^{i}\right)$ the Hilbert space of all square-integrable sections over $\Omega$ of $C^{i}$.

Let $D^{*}$ be the formal adjoint of the operator $D$ in the second sequence (1.6.20). We define the Neumann space to be the graded space $N=\oplus_{i} N^{i}$, where $N^{i}$ is the subspace of $\Gamma\left(\Omega, C^{i}\right)$ composed of all sections $u$ of $\Gamma\left(\Omega, C^{i}\right)$ satisfying the pair of boundary conditions

$$
\begin{aligned}
\langle D v, u\rangle & =\left\langle v, D^{*} u\right\rangle & & \text { for each } v \in \Gamma\left(\Omega, C^{i-1}\right), \\
\langle D v, D u\rangle & =\left\langle v, D^{*} D u\right\rangle & & \text { for each } v \in \Gamma\left(\Omega, C^{i}\right) .
\end{aligned}
$$

Define $H=\oplus_{i} H^{i}$ to be the graded subspace of $N$ which is annihilated by the laplacian $D D^{*}+D^{*} D$. Since, for $u \in N^{i}$, we have

$$
\left\langle\left(D D^{*}+D^{*} D\right) u, u\right\rangle=\|D u\|^{2}+\left\|D^{*} u\right\|^{2},
$$

we see that

$$
H^{i}=\left\{u \in N^{i} \mid D u=D^{*} u=0\right\} .
$$

Definition 2.3.1. We say that the Neumann problem is solvable on $\Omega$ for the given operator from $E$ to $F$ if, for each $i \geqq 1, H^{i}$ is closed in the Hilbert space $L_{2}\left(\Omega, C^{i}\right)$, and there exist bounded operators $N: L\left(\Omega, C^{i}\right) \rightarrow L\left(\Omega, C^{i}\right)$ mapping $\Gamma\left(\Omega, C^{i}\right)$ into $N^{i}$ such that:

(i) $N H=H N=0$, where $H: L_{2}\left(\Omega, C^{i}\right) \rightarrow H^{i}$ is the orthogonal projection;

(ii) each element $u \in \Gamma\left(\Omega, C^{i}\right)$ can be written in the form

$$
u=D D^{*} N u+D^{*} D N u+H u
$$

where, in view of (2.3.2) and (2.3.3), the terms are mutually orthogonal;

(iii) the commutation relation $D N=N D$ holds.

We remark that (iii) follows from (i) and (ii). In fact, if $u \in \Gamma\left(\Omega, C^{i}\right)$, we have

$$
\begin{aligned}
\left(D D^{*}\right. & \left.+D^{*} D\right)(D N-N D) u \\
& =D\left(D D^{*}+D^{*} D\right) N u-\left(D D^{*}+D^{*} D\right) N D u=D u-D u=0 .
\end{aligned}
$$

Since $(D N-N D) u \in N^{i+1}$ and $H(D N-N D) u=0$, we have $(D N$ $-N D) u=0$. 
By (iii), the decomposition (2.3.4) yields a cochain homotopy $I-H=D\left(D^{*} N\right)+\left(D^{*} N\right) D$, and the cohomology of the sequence

$$
0 \rightarrow \Gamma\left(\Omega, C^{0}\right) \stackrel{D}{\rightarrow} \Gamma\left(\Omega, C^{1}\right) \stackrel{D}{\rightarrow} \ldots \stackrel{D}{\rightarrow} \Gamma\left(\Omega, C^{n}\right) \rightarrow 0
$$

is therefore isomorphic to $H=\oplus H^{i}$. Since the proof of Theorem 1.6.2 applies to sections as well as to germs we see that, if the Neumann problem is solvable on $\Omega$, then the cohomology of the sequence (see (1.6.15))

$$
\Gamma(\Omega, E) \stackrel{D}{\rightarrow} \Gamma(\Omega, F) \stackrel{D_{1}}{\longrightarrow} \Gamma\left(\Omega, G_{1}\right)
$$

is isomorphic to $H^{1}$, i.e., $\operatorname{ker} D_{1} / \mathrm{im} D \cong H^{1}$. It is to be expected that, in the case where $\Omega$ is a sufficiently small disc, $H^{1}$ vanishes.

We now recall briefly some of the general Hilbert space methods which are relevant to the Neumann problem (for further details, see W. J. Sweeney [23(b)]). First we complete $N^{i}$ to a Hilbert space $B^{i}$ with the Dirichlet inner product

$$
Q(u, v)=\langle D u, D v\rangle+\left\langle D^{*} u, D^{*} v\right\rangle+\langle u, v\rangle .
$$

We define the domain of the Friedrich's extension $L$ of the laplacian $D D^{*}+D^{*} D$ to be the space of all $u \in B^{i}$ such that $u \rightarrow Q(v, u)$ extends to a bounded functional in $L_{2}\left(\Omega, C^{i}\right)$, and for such $u$ we define $f=L u$ by setting $Q(v, u)=\langle v, f\rangle+\langle v, u\rangle$. The operator $L$ is selfadjoint, and $L+I$ has an inverse which is a bounded operator from $L_{2}\left(\Omega, C^{i}\right)$ to $B^{i}$.

Let $u \in N^{i}$ and $v \in \Gamma\left(\Omega, C^{i}\right)$; then

$$
|\langle D v, u\rangle|=\left|\left\langle v, D^{*} u\right\rangle\right| \leqq\|v\| \cdot Q(u, u)^{1 / 2} .
$$

Thus, for any $u \in B^{i}, v \rightarrow\langle D v, u\rangle$ extends to a bounded functional on $L_{2}\left(\Omega, C^{i}\right)$ and hence $u$ satisfies the boundary condition (2.3.2). On the other hand, an element $u$ of $B^{i}$ does not necessarily satisfy the boundary condition (2.3.3), even if $u$ is smooth. But if the element $u$ of $\Gamma\left(\Omega, C^{i}\right)$ lies in the domain of $L$, then $u$ satisfies (2.3.3).

The ellipticity of the given operator from $E$ to $F$ implies, by Theorem 1.7.1, that the laplacian $D D^{*}+D^{*} D$ is elliptic. The following proposition is a consequence of a theorem of J. J. Kohn and L. Nirenberg [13(a)] (see Sweeney [23(b)]).

Proposition 2.3.1. Suppose that the operator from $E$ to $F$ is formally integrable and elliptic, that the boundary $\omega$ of $\Omega$ is nowhere characteristic for the laplacian $D D^{*}+D^{*} D$, and that the inclusion $B^{i} \rightarrow L_{2}\left(\Omega, C^{i}\right)$ is compact for $i \geqq 1$. Then 
(i) if $L u \in \Gamma\left(\Omega, C^{i}\right)$ where $i \geqq 1$, then $u \in \Gamma\left(\Omega, C^{i}\right)$, i.e., $u$ is smooth if Lu is;

(ii) for $i \geqq 1, H^{i}$ is finite dimensional and the range of $L$ on $\Gamma\left(\Omega, C^{i}\right)$ is closed;

(iii) the Neumann problem is solvable on $\Omega$.

The assertion (i) is a theorem of Kohn and Nirenberg (Theorem 3, $\S 1$ of $[13(a)])$. Since $H^{i}=\operatorname{ker} L$ by (i), statement (ii) is a standard result in the theory of compact operators. Finally, to prove (iii), we observe that, since $L$ is selfadjoint, $I-H$ is the orthogonal projection onto the range of $L$. For $u \in L_{2}\left(\Omega, C^{i}\right)$, we define $v=N u$ to be the unique element in the domain of $L$ satisfying $H v=0, L v=u-H u$. Then $N$ is bounded by the closed graph theorem and maps $\Gamma\left(\Omega, C^{i}\right)$ onto $N^{i}$.

Another theorem of Kohn-Nirenberg (Theorem 5, $\$ 2$ of [13(a)]) implies the following result:

Proposition 2.3.2. Suppose that the operator from $E$ to $F$ is formally integrable and elliptic, and that there is a constant $c>0$ such that, for $u \in B^{i} \cap \Gamma\left(\Omega, C^{i}\right)$ and $i \geqq 1$,

$$
Q(u, u) \geqq c \int_{\omega}|u|^{2} d s
$$

where ds denotes the volume element in the boundary $\omega$ of $\Omega$. Then the Neumann problem is solvable on $\Omega$ for the given operator.

We call (2.3.6) the Kohn-Nirenberg estimate (for $u \in B^{i} \cap \Gamma\left(\Omega, C^{i}\right)$, $i \geqq 1)$. If this estimate holds, the inclusion $B^{i} \rightarrow L_{2}\left(\Omega, C^{i}\right)$ is compact for $i \geqq 1$.

We remark that, in studying the Dirichlet norm $Q(u, u)$, we may always assume that the component of $u$ which is a section of $\Lambda^{i} T^{*} \otimes R_{m}$ is zero (see [21(b), §8]). Thus, if the hypothesis of Proposition 2.3.1, or the Kohn-Nirenberg estimate, holds on $\Omega$ for a formally integrable, elliptic operator from $E$ to $F$ of order $k$, it holds on $\Omega$ for any other formally integrable operator with the same symbol or same $g_{k}$ $\subset S^{k} T^{*} \otimes E$.

In $[23(c)]$, Sweeney considers the following elliptic complex. Let $R^{3}$ have the coordinate $(x, y, t)$, write $z=x+i y$ and let $D_{\bar{z}}=\frac{1}{2}(\partial / \partial x+i \partial / \partial y), \quad D_{y}=\partial / \partial y, \quad D_{t}=\partial / \partial t$. Take $E=R^{3} \times C^{2}, \quad F$ $=R^{3} \times C^{4}$ and $G=R^{3} \times C^{2}$, and let $D: E \rightarrow F, \varepsilon: F \rightarrow G$ be the operators defined by

$$
\begin{aligned}
D(u, v) & =\left(D_{z} v, D_{t} v, D_{3} u, D_{t} u-D_{v} v\right), \\
\varepsilon\left(w_{1}, w_{2}, w_{3}, w_{4}\right) & =\left(D_{t} w_{1}-D_{3} w_{2}, D_{t} w_{3}-D_{y} w_{1}-D_{3} w_{4}\right) .
\end{aligned}
$$


It is easy to verify that the sequence

$$
E \stackrel{\mathscr{D}}{\rightarrow} F \stackrel{\mathcal{E}}{\rightarrow} G
$$

forms an elliptic complex of sheaves and that it is exact, i.e., the inhomogeneous overdetermined system is locally solvable. We choose the obvious hermitian metrics for $E, F, G$ and take $\Omega$ to be the unit disc in $R^{3}$. Writing $w=\left(w_{1}, w_{2}, w_{3}, w_{4}\right)$, we have the Dirichlet norm

$$
Q(w)=\left(\|\varepsilon w\|^{2}+\left\|D^{*} w\right\|^{2}+\|w\|^{2}\right)^{1 / 2} .
$$

Sweeney [23(c)] shows that this Dirichlet norm is not compact on $\Omega$. In a recent letter to the author, Sweeney extends this negative result by proving the following proposition:

Proposition 2.3.3. Let $D: E \rightarrow F$ be the operator defined by the first formula (2.3.7), which is elliptic and formally integrable, and let the $C^{i}=C_{m+1}^{i}$ be defined for this operator by (1.6.3). Then the inclusion $B^{1} \rightarrow L_{2}\left(\Omega, C^{1}\right)$ is not compact for any $m \geqq 0$, where $\Omega$ is the unit disc in $R^{3}$.

Elaborations of this example show, as has been pointed out by Sweeney, that the Neumann problem is not necessarily solvable in the $L_{2}$-sense (although possibly solvable in some other sense).

Proposition 2.3.3 implies that the Kohn-Nirenberg estimate does not always hold on discs for operators with constant coefficients, and the problem arises to determine a class of operators for which it does hold on domains $\Omega$ satisfying appropriate convexity conditions. Several years ago, I. M. Singer recognized that the $\delta$-estimate implies the Kohn-Nirenberg estimate for $i=1$ on domains satisfying the required convexity requirements. Recently, MacKichan [16] extended this result by showing that the $\delta$-estimate implies the KohnNirenberg estimate for all degrees $i \geqq 1$ on the same type of domains. We shall now describe briefly how the $\delta$-estimate enters into the Kohn-Nirenberg estimate. For simplicity we assume that $\Omega$ is covered by a single coordinate $\left(x^{1}, x^{2}, \cdots, x^{n}\right)$ such that $\left(d x^{1}, d x^{2}, \cdots, d x^{n}\right)$ is an orthonormal frame for $T^{*}$; this assumption is in no way necessary but it simplifies our description.

Let $r$ be a differentiable function on $X$ such that: (i) $r(x)=0$ if and only if $x \in \omega$ (boundary of $\Omega$ ); (ii) $r(x) \geqq 0$ for $x \in \Omega$; (iii) $|d r|=1$ on $\omega$. Then $u \in B^{i} \cap \Gamma\left(\Omega, C^{i}\right)$ if and only if $u$ belongs to $\Gamma\left(\Omega, C^{i}\right)$ and satisfies the boundary condition 


$$
\sigma_{d r}(D)^{*} u=0
$$

on $\omega$. Let $u$ be an element of $\Gamma\left(\Omega, C^{i}\right)$, where $i \geqq 1$, which satisfies (2.3.8). We integrate by parts the term $\left\|D^{*} u\right\|^{2}$ in a way which has been made familiar by Kohn [12] in his solution of the Neumann problem for the operator $\bar{\partial}$ (Cauchy-Riemann operator in several complex variables). In order to formulate the result of this integration by parts, we introduce the map

$$
(1 \otimes \sigma(D))^{*}: T^{*} \otimes C^{i} \rightarrow T^{*} \otimes T^{*} \otimes C^{i-1} .
$$

Let $v$ be an element of $T^{*} \otimes C^{i}$. Then, in terms of the coordinate $\left(x^{1}, x^{2}, \cdots, x^{n}\right)$,

$$
v=\sum_{l=1}^{n} d x^{l} \otimes v_{l}
$$

where $v_{l} \in C^{i}$, and

$$
(1 \otimes \sigma(D))^{*} v=\sum_{l, m=1}^{n} d x^{l} \otimes d x^{m} \otimes A_{m}^{*} v_{l}
$$

where

$$
\sigma(D)^{*} v_{l}=\sum_{m=1}^{n} d x^{m} \otimes A_{m}^{*} v_{l}
$$

We let

$$
S: T^{*} \otimes T^{*} \otimes C^{i-1} \rightarrow T^{*} \otimes T^{*} \otimes C^{i-1}
$$

be the switching operator where

$$
S\left(d x^{l} \otimes d x^{m} \otimes c\right)=d x^{m} \otimes d x^{l} \otimes c .
$$

The integration by parts yields an inequality of the form

$$
\text { (2.3.9) } \quad c Q(u, u) \geqq\left\langle S(1 \otimes \sigma(D))^{*} d u,(1 \otimes \sigma(D))^{*} d u\right\rangle+L(u)
$$

where $c>0$ and $L(u)$ is a "Levi form." Namely, denote scalar products over the boundary by attaching a subscript $\omega$ to $\langle\cdots\rangle$, i.e., $\langle\cdots\rangle_{\omega}$ is the scalar product in the boundary and $\|\cdots\|_{\omega}$ is the corresponding norm. Then, in the case where the operator has constant coefficients, $L(u)$ has the form

$$
L(u)=\sum_{l, m=1}^{n}\left\langle\frac{\partial^{2} r}{\partial x^{l} \partial x^{m}} A_{\imath}^{*} u, A_{m}^{*} u\right\rangle_{\omega} .
$$

The convexity condition required for $\Omega$ is that there exist a constant $c^{\prime}>0$ such that 


$$
L(u) \geqq c^{\prime}\|u\|_{\omega}^{2}
$$

We suppose that (2.3.10) is satisfied; then (2.3.9) can be written in the form

$$
\|u\|_{\omega}^{2} \leqq-c\left\langle S(1 \otimes \sigma(D))^{*} d u,(1 \otimes \sigma(D))^{*} d u\right\rangle+c^{\prime} Q(u, u)
$$

where $c$ and $c^{\prime}$ are positive constants. Therefore the Kohn-Nirenberg estimate holds if there is a positive constant $c$ such that

$$
-\left\langle S(1 \otimes \sigma(D))^{*} d u,(1 \otimes \sigma(D))^{*} d u\right\rangle \leqq c Q(u, u) .
$$

Next, the inequality (2.3.11) will hold $a$ fortiorn if it holds in the point-wise sense, namely if, for every $v \in T^{*} \otimes C^{i}$, we have the pointwise inequality

$$
\begin{aligned}
-\left\langle S(1 \otimes \sigma(D))^{*} v,(1 \otimes \sigma(D))^{*} v\right\rangle_{x} & \\
& \leqq c\left\{\|\sigma(D) v\|_{x}^{2}+\left\|\sigma\left(D^{*}\right) v\right\|_{x}^{2}\right\} .
\end{aligned}
$$

In fact, suppose that (2.3.12) holds, take $v=d u$ where $u$ is an element of $\Gamma\left(\Omega, C^{i}\right)$ and integrate (2.3.12) over $\Omega$. Then we obtain (2.3.11) since $\|\sigma(D) d u\|^{2}+\left\|\sigma\left(D^{*}\right) d u\right\|^{2}$ is dominated by a constant multiple of $Q(u, u)$.

Finally, it is easily seen that (2.3.11) holds if

$$
\left\langle S(1 \otimes \sigma(D)) *_{v},(1 \otimes \sigma(D)) *\right\rangle_{x} \geqq 0
$$

for all $\sigma \in T^{*} \otimes C^{i}$ satisfying $\sigma(D) v=0$ and $\sigma\left(D^{*}\right) v=0$. We drop the condition $\sigma\left(D^{*}\right) v=0$. Then we have the main result (see Mackichan [14]):

THeOREM 2.3.1. The inequality (2.3.13) for all $v \in g_{1}^{i}=\operatorname{ker} \sigma\left(D^{i}\right)$ is equivalent to the estimate of Proposition 2.2.2, namely

$$
\|\delta x\|^{2} \geqq \frac{1}{2}\|x\|^{2}
$$

for all $\chi \in\left(T^{*} \otimes g_{1}^{i}\right) \cap$ ker $\delta^{*}$. Hence, if the given operator from $E$ to $F$ satisfies the $\delta$-estimate, then the Kohn-Nirenberg estimate holds on $\Omega$ for all $u \in \Gamma\left(\Omega, C^{i}\right), i \geqq 1$, satisfying (2.3.8), provided that $\Omega$ satisfies (2.3.10).

Let $v \in T^{*} \otimes C^{i}$; then, in terms of the coordinate, $v=\sum d x^{l} \otimes v_{l}$ where $v_{l} \in C^{i}$ and we have 


$$
\left\langle S(1 \otimes \sigma(D))^{*} v,(1 \otimes \sigma(D))^{*} v\right\rangle=\sum_{l, m=1}^{n}\left\langle A_{l}^{*} v_{m}, A_{m}^{*} v_{l}\right\rangle .
$$

Thus the $\delta$-estimate implies the inequality

$$
\sum_{l, m=1}^{n}\left\langle A_{l}^{*} v_{m}, A_{m}^{*} v_{l}\right\rangle \geqq 0
$$

for all $v=\sum d x^{i} \otimes v_{l} \in g_{1}^{i}=\operatorname{ker} \sigma\left(D^{i}\right)$. The form of this inequality (apart from sign) is remarkably similar to (2.2.2).

The following recent result of Sweeney complements Theorem 2.3.1.

THEOREM 2.3.2. Suppose that the given operator from $E$ to $F$ is elliptic and satisfies the $\delta$-estimate. Then, on a sufficiently small coordinate disc (defined in terms of a fixed coordinate system), the harmonic space of the Neumann problem vanishes in positive degrees.

It follows from Proposition 2.3.2, Theorems 2.3.1 and 2.3.2 that, for an elliptic operator satisfying the $\delta$-estimate, the second sequence attached to the operator is exact at degree 1 and hence, by Theorem 1.6.2, the overdetermined inhomogeneous system is locally solvable.

The $\bar{\partial}$-operator (Cauchy-Riemann operator in several complex variables) is elliptic and satisfies the $\delta$-estimate.

Finally it might be worthwhile to determine the class of operators deformable into those satisfying the $\delta$-estimate by deformations leaving the base manifold fixed. Clearly Sweeney's example $(2.3,7)$ belongs to this class.

\section{REFERENCES}

1. R. BotT, Notes on the Spencer resolution, Harvard University, Cambridge, Mass., 1963.

2. C. Butrin, Existence of a homotopy operator for Spencer's sequence in the analytic case, Pacific J. Math. 21 (1967), 219-240.

3. H. CARTAN, Familles d'espaces complexes et fondements de la géométrie analytique, Séminaire Henri Cartan, 1961/62, Secrétariat Mathématique, Paris, 1963.

4. B. CENKL, Vanishing theorem for an elliptic differential operator, J. Differential Geometry 1 (1967), 381-418.

5. L. Ehrenpreis, V. W. Gutllemin and S. Sternberg, On Spencer's estimate for $\delta$-Poincaré, Ann. of Math. (2) 82 (1965), 128-138.

6. H. Goldschmidt, (a) Existence theorems for analytic linear partial differential equations, Ann. of Math. (2) 86 (1967), 246-270.

(b) Integrability criteria for systems of non-linear partial differential equations, J. Differential Geometry 1 (1967), 269-307.

(c) A prolongation theorem for systems of linear partial differential equations. I: A conjecture of E. Cartan, Ann. Sci. Ecole Norm. Sup. 1(1968), 417-444.

(d) Prolongations of linear partial differential equations. II: Inhomogeneous equations, Ann. Sci. Ecole Norm. Sup. (to appear).

(e) Estimates for analytic partial differential equations (to appear). 
7. A. Grothendieck, Éléments de gêométrie algébrique. IV, Inst. Hautes Etudes Sci. Publ. Math. No. 24, 1965, pp. 231.

8. V. GuILLEMIN, Some algebraic results concerning the characteristics of overdetermined partial differential equations, Amer. J. Math. 90 (1968), 270-284.

9. V. GUILLEMIN and M. KURANISHI, Some algebraic results concerning involutive subspaces, Amer. J. Math. (to appear).

10. V. Guillemin, D. QUillen and S. STERnBerg, The classification of the complex primitive infinite pseudogroups, Proc. Nat. Acad. Sci. U.S.A. 55 (1966), 687-690.

11. L. Hörmander, (a) Linear partial differential operators, Die Grundlehren der Math. Wiss., Band 116, Academic Press, New York and Springer-Verlag, Berlin, 1963; Russian transl., "Mir", Moscow, 1965.

(b) Pseudo-differential operators, Comm. Pure Appl. Math. 18 (1965), 501-517.

(c) Pseudo-differential operators and non-elliptic boundary problems, Ann. of Math. (2) 83 (1966), 129-209.

12. J. J. KоHN, (a) Harmonic integrals on strongly pseudo-convex manifolds. I, Ann. of Math. (2) 78 (1963), 112-148.

(b) Harmonic integrals on strongly pseudo-convex manifolds. II, Ann. of Math. (2) 79 (1964), 450-472.

(c) Boundaries of complex manifolds, Proc. Conf. Complex Analysis (Minneapolis, 1964), Springer-Verlag, Berlin, 1965.

13. J. J. KoHN and L. Nirenberg, (a) Non-coercive boundary value problems, Comm. Pure Appl. Math. 18 (1965), 443-492.

(b) An algebra of pseudo-differential operators, Comm. Pure Appl. Math. 18 (1965), 269-305.

14. M. KuRANISHI, (a) On É. Cartan's prolongation theorem of exterior differential systems, Amer. J. Math. 79 (1957), 1-47.

(b) Sheaves defined by differential equations and application to deformation theory of pseudo-group structures, Amer. J. Math. 86 (1964), 379-391.

15. H. LEwy, An example of a smooth linear partial differential equation without solution, Ann. of Math. (2) 66 (1957), 155-158.

16. B. MacKichan, A generalization to overdetermined systems of the notion of diagonal operators, Thesis, Stanford University, Stanford, Calif., 1968 (to appear).

17. B. Malgrange, (a) Cohomologie de Spencer (d'après Quillen), Secrétariat Mathématique d'Orsay, 1966.

(b) Théorie analytique des équations différentielles, Séminaire Bourbaki, no. 329, 1966-67, Secrétariat Mathématique.

18. C. B. MORREY, (a) The analytic embedding of abstract real-analytic manifolds, Ann. of Math. (2) 68 (1958), 159-201.

(b) The $\partial$-Neumann problem on strongly pseudo-convex manifolds, Outlines Joint Sympos. Partial Differential Equations, (Novosibirsk, 1963), Acad. Sci. USSR Siberian Branch, Moscow, 1963, pp. 171-178.

19. D. G. Quillen, Formal properties of over-determined systems of linear partial differential equations, Thesis, Harvard University, Cambridge, Mass., 1964 (unpublished).

20. J.-P. Serre, Faisceaux algebriques coherents, Ann. of Math. (2) 61 (1955), 197-278; Russian transl., Chapter Xin Fiber spaces and their applications, IL, Moscow, 1958.

21. D. C. Spencer, (a) Deformation of structures on manifolds defined by transitive, continuous pseudogroups. I, II, Ann. of Math. (2) 76 (1962), 306-445. 
(b) Deformation of structures on manifolds defined by transitive, continuous pseudogroups. III, Ann. of Math. (2) 81 (1965), 389-450.

(c) A type of formal exterior differentiation associated with pseudogroups, Scripta Math. 26 (1961), 101-106.

22. S. Sternberg, Partial differential equations, Lectures at the University of Pennsylvania, January-February, 1967 (polycopied).

23. W. J. SweEnEy, (a) The $\delta$-Poincaré estimate, Pacific J. Math. 20 (1967), 559570.

(b) The D-Neumann problem, Acta Math. 120 (1968), 223-277.

(c) A non-compact Dirichlet norm, Proc. Nat. Acad. Sci. U.S.A. 58 (1967), 21932195.

24. NGô VAN QUÊ, Du prolongement des espaces fubrés et des structures infinitésimales, Ann. Inst. Fourier (Grenoble) 17 (1967), 157-223.

Princeton University, Princeton, New Jersey 08540 